\title{
Paediatric epilepsy surgery in the posterior cortex: a study of 62 cases
}

\author{
Alexandra Liava ${ }^{1,2}$, Roberto Mai ${ }^{1}$, Laura Tassi ${ }^{1}$, Massimo \\ Cossu $^{1}$, Ivana Sartori ${ }^{1}$, Lino Nobili ${ }^{1}$, Giorgio Lo Russo ${ }^{1}$, \\ Stefano Francione ${ }^{1}$ \\ 1 "Claudio Munari" Epilepsy Surgery Centre, Niguarda Ca' Granda Hospital \\ ${ }^{2}$ Neuroscience Department, University of Milan-Bicocca, Milan, Italy
}

Received September 13, 2013; Accepted February 03, 2014

ABSTRACT - Past surgical series have emphasized the diagnostic complexity of posterior cortex epilepsy (PCE). Available data are sparse, especially in children, and most published series report a high number of surgical failures and post-operative neurological deficits. In this article, we present a paediatric cohort of 62 children who underwent surgery for pharmaco-resistant PCE before the age of 16 years with a mean postoperative follow-up of 6.94 years (range: 2-16). Mean age at epilepsy onset was 3.2 years and 28 children ( $45 \%$ ) had onset before 1 year of age. The mean age at surgery was 7.9 years (range: $1-16$ ). Daily seizures were present in $63 \%$ of children. MRI was positive in 58 cases $(93.5 \%)$ and invasive stereo-EEG was judged mandatory in $24 / 62(39 \%)$ of patients. Surgery was confined to the parietal lobe in 11 children, the occipital lobe in 8 , the occipito-parietal region in four, the occipito-temporal region in 18, and involved both the temporal and parietal lobes in the remaining 21. Following surgery, 53 subjects $(85.5 \%)$ remained seizure-free and among those who underwent a SEEG procedure, $75 \%$ achieved seizure freedom. Focal cortical dysplasia was the most frequent histopathological diagnosis $(50 \%)$, followed by tumoural $(24 \%)$ and gliotic lesions (14.5\%). An older age at epilepsy onset, the presence of a rather restricted epileptogenic area, and a complete resection of the epileptogenic zone were predictive of a favourable surgical outcome. These results demonstrate that a good surgical outcome is possible in children with pharmaco-resistant PCE. Accurate analysis of the chronology of ictal semiology and electrophysiological features, viewed in the context of the complete electroclinical pattern, provides a topographical orientation for PCE and, together with the presence of a lesion detectable on imaging, may improve the rate of surgical success of PCE at paediatric age.

Correspondence:

Alexandra Liava

"Claudio Munari" Epilepsy Surgery

Centre, Niguarda Hospital,

Neuroscience Department, University of Milan-Bicocca,

Piazza Ospedale Maggiore 3,

20162 Milan, Italy

<alexandra_liava@yahoo.it>

Key words: occipital epilepsy, parietal epilepsy, epilepsy surgery, extratemporal epilepsy, children

Epilepsies originating from the posterior cortex, namely the parietooccipital lobes and the occipital border of the temporal lobe, account for the minority of focal epilepsies (Boesebeck et al., 2002), therefore, epilepsy surgery in the posterior cortex has been less extensively 
examined in the literature, relative to that for temporal or frontal lobe epilepsies.

Most studies emphasize the difficulty in delineating the precise localisation of a posterior epileptogenic zone (EZ: the site of origin and of primary propagation of ictal discharges; Bancaud et al., 1970; Kahane et al., 2006) because of rather non-specific clinical seizure patterns (Bancaud, 1969; Williamson et al., 1992; Boesebeck et al., 2002; Bartolomei et al., 2011). Ictal semiology in posterior cortex epilepsy (PCE) will vary according to the discharge pathway and can falsely localise to the anterior cortical regions. This difficulty is even more relevant in paediatric epilepsy, as EEG patterns are frequently generalised and the subjective and objective chronology of ictal semiology is difficult to obtain.

Paediatric series of PCE surgery are rare (Sinclair et al., 2005Q1; Mohamed et al., 2011; Ibrahim et al., 2012); children are frequently included in surgical series of adult patients undergoing either occipital lobe epilepsy (OLE) surgery (Salanova et al., 1992; Williamson et al., 1992; Caicoya et al., 2007; Binder et al., 2008; Tandon et al., 2009), parietal lobe epilepsy (PLE) surgery (Binder et al., 2009) or PCE surgery (Dalmagro et al., 2005; Yu et al., 2009 Q2; Jehi et al., 2009). Therefore, the aim of this study was to characterise the anatomo-electroclinical features and to describe the surgical outcome of an exclusively paediatric patient population, surgically treated for pharmaco-resistant PCE.

\section{Patients and methods}

The patients included in this study were part of a paediatric population of 312 consecutive subjects who underwent epilepsy surgery between May 1996 and April 2011 at the "Claudio Munari" Epilepsy Surgery Centre. The patients were identified from a prospective epilepsy surgery database on the basis of the following inclusion criteria: (i) tailored surgery performed for focal pharmaco-resistant epilepsy; (ii) age at surgery of less than 16 years; (iii) at least 24 months of post-operative follow-up; (iv) surgical resection including the occipital and the parietal lobe, separately, and in combination and association with the temporal lobe.

Sixty-two children (24 females) were identified; these represented the $19.8 \%(62 / 312)$ of the patients who underwent tailored epilepsy surgery at paediatric age. Surgery was confined to the parietal lobe (PL) in 11 children $(17.8 \%)$ and to the occipital lobe $(\mathrm{OL})$ in 8 $(13 \%)$. Four children $(6.4 \%)$ underwent a tailored resection in the occipito-parietal region and in $18(29 \%)$, the resection involved the occipito-temporal region. In the remaining 21 subjects (33.8\%), the excision involved the occipito-temporo-parietal (OTP) area.
For all patients, the pre-surgical evaluation protocol included: (i) detailed history-taking with accurate analysis of the chronology of semiology of the habitual seizure pattern; (ii) neurological and neuropsychological examination with standardised tests according to age; (iii) prolonged scalp video-EEG monitoring with scalp electrodes placed according to the international 10-20 system; and (iv) high-resolution MRI with images acquired parallel to and perpendicular to the anteroposterior commissural line, with coronal sequences localised over the area of seizure generation, as indicated by the electroclinical data (Colombo et al., 2003), and, when necessary, functional imaging (DTI, fMRI, and PET).

In cases where the data obtained by non-invasive presurgical evaluation were discordant, or when MRI was non-informative, or when highly functional regions were located immediately close to the presumed EZ, stereoelectroencephalography (SEEG) monitoring was performed. The implantation strategy was based on individual electroclinical hypothesis, aiming to explore the areas putatively involved in seizure onset and early propagation, according to the Bancaud and Talairach method (Cossu et al., 2006; Cossu et al., 2012; Cardinale et al., 2013). Following electrode implantation, electrical bipolar cortical stimulations of two adjacent contacts were carried out at both low (LF: $1 \mathrm{~Hz}$, pulse width: $2 \mathrm{~ms}$, during $30 \mathrm{~s}$ ) and high frequency (HF: $50 \mathrm{~Hz}$, pulse width: $1 \mathrm{~ms}$, during $5 \mathrm{~s}$ ) in order to define the eloquent regions such as the motor, language, and visual cortices, and to reproduce the patient's ictal symptoms and signs.

Six months after surgery, all subjects had a first follow-up visit with EEG, MRI, and neuropsychological evaluation; further follow-up was undertaken annually, up to at least five years after surgery. Postoperative outcome was documented according to Engel's classification (Engel et al., 1993) and in this series refers to the last available follow-up visit.

Ictal clinical symptoms were assessed by analysing video-recorded seizures, obtained during VEEG and/or SEEG monitoring, in 56 patients. In the remaining 6 , video was not available and we only considered seizures that were clearly described as typical by the patient or the patient's family. The seizure semiology was analysed according to the chronology of ictal and postictal signs, and classified according to the ILAE glossary (Blume et al., 2001 Q3). Ictal EEG onset was defined by the occurrence of a low-voltage fast (beta/gamma) activity or a well-localised flattening, by the disappearance of well-localised interictal EEG abnormalities, or (in the absence of the three previous patterns) by the occurrence of a rhythmic discharge. Ictal electrophysiological patterns, as well as interictal activity, were classified according to their: lateralisation (right, left, or bilateral), extent (localised: location 
at no more than two contiguous electrodes; regional: involvement of multiple electrodes over the posterior quadrant; or generalised: involvement of multiple electrodes over both cerebral hemispheres), and morphology (spikes, spike-waves, slow-band: delta, theta, and fast-band activity).

Patients were classified as MRI-positive when the pre-operative MRI demonstrated a discrete, even uncertain, lesion which was possibly coherent with the electroclinical picture, and MRI-negative when MRI was totally unremarkable.

The surgical resection of the EZ was judged complete when the entire epileptogenic area, defined by the anatomo-electro-clinical features of each single patient, was removed. In lesional cases, this did not necessarily imply the complete resection of the MRIdetectable lesion.

\section{Results}

The 62 identified PCE patients were analysed as a whole (table 1) and then subcategorised into four groups (table 2): (i) patients who benefited from a tailored excision strictly within the limits of the parietal lobe, namely the pure-parietal epilepsy group (pure-PLE; 11 children); (ii) patients who underwent a resection confined within the limits of the occipital lobe, the pure-occipital epilepsy group (pure-OLE; 8 children); (iii) children who benefited from a resection involving primarily the occipital lobe overlapping the parietal and/or temporal lobe (O+ group; 26 children); and (iv) subjects who underwent a vast multilobar resection (extended-OTP group; 17 children).

Detailed anatomo-electro-clinical features of each patient are summarised in the supplementary tables S1-4.

\section{Patient characteristics}

All PCE patients were diagnosed with sporadic, nonfamilial epilepsy. In 11 subjects, there was a history of maternal pregnancy complication characterised by a threat of miscarriage. Eight patients had a personal history of perinatal hypoxic-ischaemic insult. Two subjects suffered from hypoxia during cardiac malformation surgery and one child suffered from postnatal viral encephalitis. The remaining patients had an unremarkable personal history. Neurological presurgical examination was strictly normal in 34 subjects. Nine subjects presented with motor impairment, characterised by hemiparesis of variable severity in eight and dyspraxia in one subject.

Deficits in oculomotor functions were observed exclusively in children who underwent a resection involving
Table 1. Anatomo-electro-clinical features of the global population (62 patients)*.

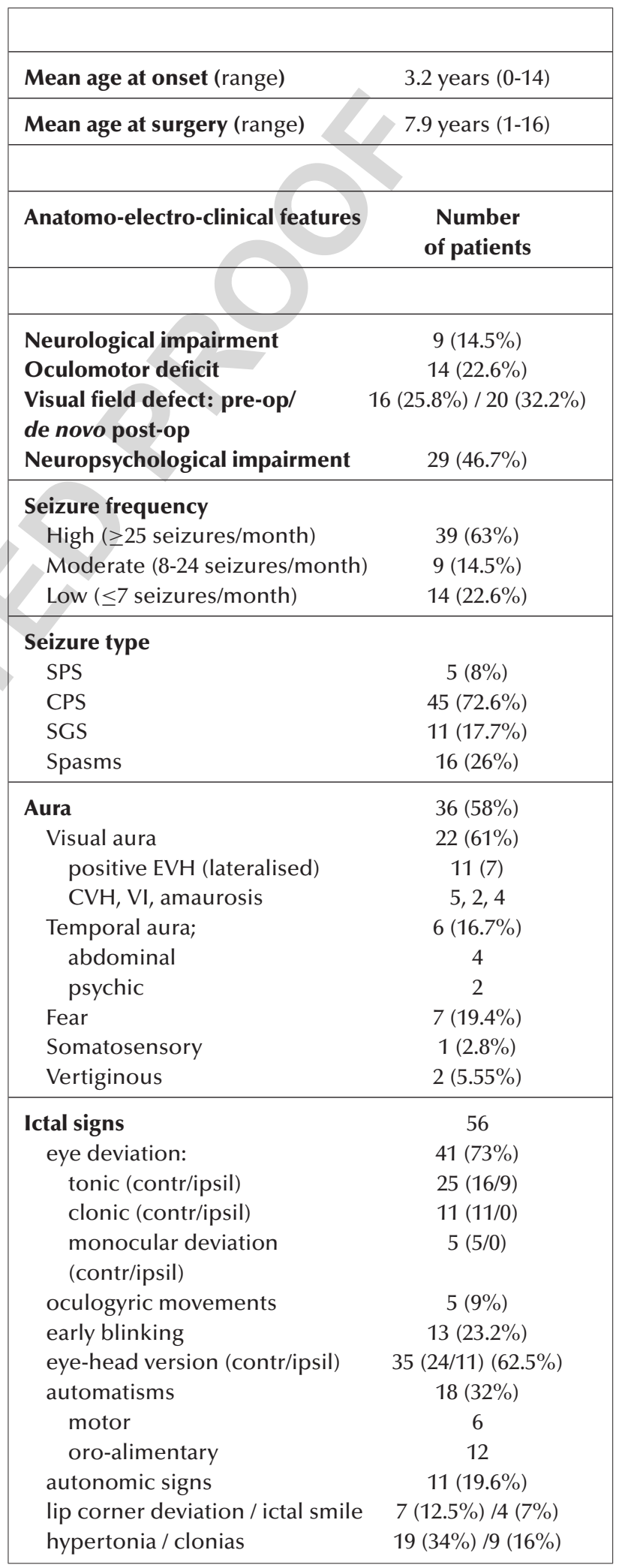


Table 1. (Continued)

\begin{tabular}{|c|c|}
\hline Asymmetric EEG background & $44(71 \%)$ \\
\hline Interictal EEG & 62 \\
\hline Localising & $22(35.5 \%)$ \\
\hline Regional & $25(40.3 \%)$ \\
\hline Falsely localising & $6(2 \mathrm{~F}, 3 \mathrm{FT}, 1 \mathrm{~T})(9.7 \%)$ \\
\hline Lateralising only & $3(4.8 \%)$ \\
\hline Falsely lateralising & $6(9.7 \%)$ \\
\hline Ictal EEG & 56 \\
\hline Localising & $21(37.5 \%)$ \\
\hline Regional & $18(32 \%)$ \\
\hline Falsely localising & $7(\mathrm{~F})(12.5 \%)$ \\
\hline Lateralising only & $1(1.8 \%)$ \\
\hline Falsely lateralising & $3(5.35 \%)$ \\
\hline Bi-hemispheric/diffuse & $6(10.7 \%)$ \\
\hline MRI-positive & $58(93.5 \%)$ \\
\hline Lobar/sublobar & $5 / 12$ \\
\hline Bilobar & 25 \\
\hline Multilobar & 16 \\
\hline \multicolumn{2}{|l|}{ Engel class } \\
\hline I (Ia, Ib, Ic, Id $)$ & $53(52,0,0,1)(85.5 \%)$ \\
\hline Ila & $2(3.2 \%$ \\
\hline IIla & $4(6.5 \%)$ \\
\hline IVa & $3(4.8 \%)$ \\
\hline SEEG & $24(38.7 \%)$ \\
\hline SEEG Seizure-free & $18(75 \%)$ \\
\hline \multicolumn{2}{|l|}{ Histology } \\
\hline FCD & $31(50 \%)$ \\
\hline Tumours & $15(24.2 \%)$ \\
\hline Gliosis & $9(14.5 \%)$ \\
\hline Others & $7(11.3 \%)$ \\
\hline
\end{tabular}

*More than one sign could coexist in the same individual. op: operative; SPS: simple focal seizures; CPS: complex focal seizures; SGS: secondary generalised seizures; EVH: elementary visual hallucinations; CVH: complex visual hallucinations; VI: visual illusions; ipsil: ipsilateral; contr: contralateral.

the OL (51/62 children); $27.4 \%$ (14/51 subjects) presented with a pre-operative oculomotor deficit, and in 11 of these 14 patients, this was characterised by a convergent strabismus of the eye, contralateral to the epileptogenic side. Moreover, a pre-operative visual field deficit concerned $29.4 \%$ of the same group (15/51) in the form of homonymous hemianopsia $(\mathrm{HH})$ in 8 and homonymous quadrantanopsia $(\mathrm{HQ})$ in 7 cases. One child in the pure-PLE group $(9 \% ; 1 / 11)$ also presented with an inferior quadrantic defect. In 14 patients, a formal perimetry evaluation was not available due to non-cooperation during visual field testing.
Four children presented with global cognitive impairment and 25 exhibited neuropsychological deficits, mostly consisting of deficits in attention, visuo-spatial and executive functions, and verbal fluency; 5 children presented with behavioural problems.

Mean age at epilepsy onset was 3.2 years (0-14 years) and mean age at surgery was 7.9 (1-16 years); $45 \%$ of children (28/62) had seizure onset within the first year of life.

\section{Ictal semiology}

Forty-five children $(73 \%)$ presented with complex partial seizures (CPS), 16 subjects $(26 \%)$ presented with spasms (figure 1), 8 of whom also had CPS, 11 children $(18 \%)$ had secondary generalised seizures (SGS), and $5(8 \%)$ had simple focal seizures. A large proportion of children had a high seizure frequency and $63 \%$ presented daily seizures; in seven patients, clinical evolution was complicated by status epilepticus.

Prevalent ictal semiology consisted of subjective visual symptoms followed by oculomotor signs, after which children exhibited either an axial deviation of the head followed by a hemiclonic or adversive seizure (frontal pattern: 19 subjects; $30.6 \%$ ) or autonomic/vegetative manifestations and oro-alimentary automatisms (temporal pattern: 34 patients; $54.8 \%$ ) (figure 2). The former pattern prevailed among the pure-PLE subjects $(60 \%)$, while the latter was more frequent among the children who underwent an excision involving the OL $(49 \%)$.

Visual subjective symptoms were experienced by 22 subjects, in the form of positive elementary visual hallucinations (EVH) in 11 cases, amaurosis in 4 cases, and complex visual hallucinations $(\mathrm{CVH})$ and visual illusions ( $\mathrm{VI})$ in 7 cases. The elementary positive visual aura was lateralised in 7 cases $(7 / 11 ; 63.6 \%)$. An initial abdominal discomfort was encountered in 4 subjects $(12.5 \%), 7$ children reported fear, 2 reported a sensation of unreality, 2 reported a vertiginous sensation, and 1 reported focal paresthesias.

An ictal VEEG was available in 56 cases; 268 seizures (mean: 4.8 seizures/child) were recorded and analysed. The leading ictal sign consisted of oculomotor manifestations or blinking in 47 children (84\%): clonic eye deviation (epileptic nystagmus) was exhibited in 11 patients, in all cases contralateral to the side of the excision; tonic eye deviation in 25 cases, in 16 cases contralateral to the side of the excision; a monocular deviation in 5 children, consisting of convergent strabismus of the eye, contralateral to the epileptogenic side; and early blinking in 13 children, associated with oculoclonic movements in 6 patients, with tonic eye deviation in 2 and staring in 2 cases. Oculogyric/opsoclonic movements, consisting of chaotic, involuntary, and multidirectional saccades without 
Table 2. Anatomo-electro-clinical characteristics of the groups, as defined by the topography of the resection*.

\begin{tabular}{|c|c|c|c|c|}
\hline Mean age at onset (years) & $6(1-13)$ & $3.27(0-14)$ & $1.45(0-5)$ & $3.2(0-9)$ \\
\hline \multirow[t]{3}{*}{ Mean age at surgery (years) } & $8.5(2-14)$ & $9.58(2-16)$ & $5.9(2-16)$ & $7.27(1-12)$ \\
\hline & \multicolumn{4}{|c|}{ Number of patients } \\
\hline & $\begin{array}{l}\text { Pure-OLE } \\
\text { (8 patients) }\end{array}$ & $\begin{array}{c}\text { O+ } \\
\text { (26 patients) }\end{array}$ & $\begin{array}{c}\text { Extended OTP } \\
\text { (17 patients) }\end{array}$ & $\begin{array}{c}\text { Pure-PLE } \\
\text { (11 patients) }\end{array}$ \\
\hline Personal antecedents & $1(12.5 \%)$ & $2(7.7 \%)$ & $5(29.4 \%)$ & $3(27 \%)$ \\
\hline $\begin{array}{l}\text { Visual field deficit } \\
\text { pre-op/de novo post-op } \\
\text { HH } \\
\text { pre-op/de novo post-op }\end{array}$ & $5(62.5 \%) / 3(37.5 \%)$ & $\begin{array}{l}5(19.23 \%) / 12(46 \%) \\
2(7.7 \%) / 5(19.23 \%)\end{array}$ & $\begin{array}{l}5(29.4 \%) / 6(35.3 \%) \\
5(29.4 \%) / 6(35.3 \%)\end{array}$ & $1(9 \%) / 0$ \\
\hline Oculomotor deficit & $2(25 \%)$ & $3(11.5 \%)$ & $9(53 \%)$ & 0 \\
\hline Neurological impairment & 0 & $2(7.7 \%)$ & $3(17.6 \%)$ & $4(36.4 \%)$ \\
\hline Npsy impairment & $3(37.5 \%)$ & $10(38.5 \%)$ & $11(64.7 \%)$ & $5(45.5 \%)$ \\
\hline \multicolumn{5}{|l|}{ Seizure type } \\
\hline SPS & 0 & $2(7.7 \%)$ & $1(5.9 \%)$ & $2(18.2 \%)$ \\
\hline CPS & $7(87.5 \%)$ & $21(80.7 \%)$ & $9(53 \%)$ & $8(72.7 \%)$ \\
\hline Spasms & $1(12.5 \%)$ & $5(19.2 \%)$ & $8(47 \%)$ & $2(18.2 \%)$ \\
\hline SGS & $2(25 \%)$ & $2(7.7 \%)$ & $1(5.9 \%)$ & $6(54.5 \%)$ \\
\hline Status & 0 & $1(3.84 \%)$ & $6(35.3 \%)$ & 0 \\
\hline Aura & $6(75 \%)$ & $16(61.5 \%)$ & $10(58.8 \%)$ & $4(36.4 \%)$ \\
\hline Visual aura: & $6(100 \%)$ & $11(68.7 \%)$ & $5(50 \%)$ & 0 \\
\hline positive EVH (lateralised) & $5(4)$ & $5(3)$ & $1(0)$ & \\
\hline $\mathrm{CVH}, \mathrm{VI}$ & 0 & 2,2 & 0,3 & \\
\hline amaurosis & 1 & 2 & 1 & \\
\hline Psychic aura & 0 & $2(12.5 \%)$ & $1(10 \%)$ & 0 \\
\hline Abdominal aura & 0 & $3(18.7 \%)$ & $1(10 \%)$ & 0 \\
\hline Fear & 0 & $2(12.5 \%)$ & $3(30 \%)$ & $2(50 \%)$ \\
\hline Somatosensory aura & 0 & 0 & 0 & $1(25 \%)$ \\
\hline Vertiginous sensation & 0 & $1(6.2 \%)$ & 0 & $1(25 \%)$ \\
\hline Objective ictal signs & 5 & 24 & 17 & 10 \\
\hline Initial eye deviation & $4(60 \%)$ & $21(87.5 \%)$ & $12(70.6 \%)$ & $4(40 \%)$ \\
\hline tonic (contr/ipsil) & $2(1 / 1)$ & $16(10 / 6)$ & $8(7 / 1)$ & $4(3 / 1)$ \\
\hline clonic (contr/ipsil) & $2(2 / 0)$ & $5(5 / 0)$ & $4(4 / 0)$ & 0 \\
\hline Head dev (contr/ipsil) & 0 & $2(2 / 0)$ & $5(4 / 1)$ & $2(1 / 1)$ \\
\hline eye $\rightarrow$ head dev (contr/ipsil) & $4(3 / 1)$ & $15(9 / 6)$ & $4(3 / 1)$ & $3(30 \%)(2 / 1)$ \\
\hline Oculogyric movement & $2(40 \%)$ & $1(4.2 \%)$ & $2(11.8 \%)$ & 0 \\
\hline Early Blinking & $1(20 \%)$ & $7(29 \%)$ & $5(29.4 \%)$ & 0 \\
\hline Motor contralateral & $2(40 \%)$ & $10(42 \%)$ & $9(53 \%)$ & $7(70 \%)$ \\
\hline hypertonic/dystonic & $2(40 \%)$ & $7(29 \%)$ & $7(41.2 \%)$ & $3(30 \%)$ \\
\hline clonic & 0 & $3(12.5 \%)$ & $2(11.8 \%)$ & $4(40 \%)$ \\
\hline Automatisms & $2(40 \%)$ & $7(29 \%)$ & $5(29.4 \%)$ & $4(40 \%)$ \\
\hline motor/oral & $0 / 2(40 \%)$ & $1(4.2 \%) / 6(25 \%)$ & $2(11.8 \%) / 3(17.6 \%)$ & $3(30 \%) / 1(10 \%)$ \\
\hline Autonomic signs & $2(40 \%)$ & $6(25 \%)$ & $2(11.8 \%)$ & $1(10 \%)$ \\
\hline Lip corner dev, ipsil & 0 & $5(20.8 \%), 1(4.2 \%)$ & 0 & $2(20 \%), 0$ \\
\hline Ictal smile & $1(20 \%)$ & 0 & $2(11.8 \%)$ & $1(10 \%)$ \\
\hline
\end{tabular}


A. Liava, et al.

Table 2. (Continued)

\begin{tabular}{|c|c|c|c|c|}
\hline Asymmetric EEG background & $8(100 \%)$ & $16(61.5 \%)$ & $16(94 \%)$ & $4(36.3 \%)$ \\
\hline Interictal EEG & 8 & 26 & 17 & 11 \\
\hline Localising & $3(37.5 \%)$ & $8(30.7 \%)$ & $7(41.2 \%)$ & $4(36.3 \%)$ \\
\hline Regional & $4(50 \%)$ & $10(38.5 \%)$ & $7(41.2 \%)$ & $4(36.3 \%)$ \\
\hline Falsely localising & 0 & $3(1 \mathrm{~F}, 2 \mathrm{FT})(11.5 \%)$ & $1(\mathrm{~F})(5.9 \%)$ & $2(1 \mathrm{~T}, 1 \mathrm{FT})(18.2 \%)$ \\
\hline Lateralising only & 0 & 0 & $2(11.8 \%)$ & $1(9 \%)$ \\
\hline Falsely lateralising & $1(12.5 \%)$ & $5(19.2 \%)$ & 0 & 0 \\
\hline Ictal EEG & 5 & 24 & 17 & 10 \\
\hline Localising & $2(40 \%)$ & $7(29 \%)$ & $9(53 \%)$ & $3(30 \%)$ \\
\hline Regional & $1(20 \%)$ & $7(29 \%)$ & $5(29.4 \%)$ & $5(50 \%)$ \\
\hline Falsely localising & 0 & 5 (F) $(20.8 \%)$ & $2(\mathrm{~F})(11.8 \%)$ & 0 \\
\hline Lateralising only & 0 & 0 & 0 & $1(10 \%)$ \\
\hline Falsely lateralising & $2(40 \%)$ & $1(4 \%)$ & 0 & 0 \\
\hline Bi-hemispheric/diffuse & 0 & $4(16.6 \%)$ & $1(5.9 \%)$ & $1(10 \%)$ \\
\hline \multicolumn{5}{|l|}{ MRI } \\
\hline Negative & 0 & 0 & 2 & 1 \\
\hline Uncertain & 0 & 0 & 0 & 2 \\
\hline Positive & 8 & 26 & 15 & 8 \\
\hline sublobar & 3 & 4 & 0 & 4 \\
\hline lobar & 1 & 2 & 0 & 2 \\
\hline bilobar & 4 & 17 & 3 & 1 \\
\hline multilobar & 0 & 3 & 12 & 1 \\
\hline Seizure-free & $8(100 \%)$ & $24(92.3 \%)$ & $12(70.6 \%)$ & $9(81.8 \%)$ \\
\hline SEEG & 0 & $12(46 \%)$ & $6(35.3 \%)$ & $6(54.5 \%)$ \\
\hline SEEG Seizure-free & - & $11(89 \%)$ & $3(50 \%)$ & $4(66.6 \%)$ \\
\hline \multicolumn{5}{|l|}{ Engel class } \\
\hline I (Ia, Ib, Ic, Id $)$ & $8(8,0,0,0)(100 \%)$ & $24(23,0,0,1)(92.3 \%)$ & $12(12,0,0,0)(70.6 \%)$ & $9(9,0,0,0)(82 \%)$ \\
\hline Ila & 0 & 0 & $1(5.9 \%)$ & $1(9 \%)$ \\
\hline IIIa & 0 & $1(3.8 \%)$ & $2(11.8 \%)$ & 0 \\
\hline IVa & 0 & $1(3.8 \%)$ & $2(11.8 \%)$ & $1(9 \%)$ \\
\hline FCD & $1(12.5 \%)$ & $15(57.7 \%)$ & $11(65 \%)$ & $4(36.3 \%)$ \\
\hline Gliosis & 0 & $3(11.5 \%)$ & $5(29.5 \%)$ & $1(9 \%)$ \\
\hline Tumours/DNTs & $5(62.5 \%)$ & $6(23 \%)$ & 0 & $4(36.3 \%)$ \\
\hline Others & $2(25 \%)$ & $1(3.8 \%)$ & $2(11.7 \%)$ & $2(18 \%)$ \\
\hline
\end{tabular}

*More than one sign could coexist in the same individual.

CPS: complex partial seizures; Npsy: neuropsychological; op: operative; HH: homonymous hemianopsia; SPS: simple focal seizures; SGS: secondary generalised seizures; $\mathrm{EVH}$ : elementary visual hallucinations; $\mathrm{CVH}$ : complex visual hallucinations; $\mathrm{VI}$ : visual illusions; dev: deviation; ipsil: ipsilateral; contr: contralateral.

inter-saccadic intervals, were exhibited in 5 children, associated with a sensation of eye movement in 2 cases. In 26 cases, eye deviation was followed by a, more or less, slow consensual head version. Automatisms were observed in 18 children (32\%); motor automatisms prevailed among the pure-PLE group $(30 \%)$ whereas oro-alimentary automatisms prevailed among the children who underwent an excision involving the $\mathrm{OL}$ $(21.5 \%)$. Similarly, a focal motor contralateral ictal sign (hypertonic/dystonic or clonic) was more frequent among the pure-PLE group (70\%) compared with the remaining subjects $(41 \%)$.

\section{Electrophysiological features}

In all cases but one, long-term scalp EEG, either interictal or ictal, was correctly lateralising. Background activity was asymmetric in $71 \%$ of cases, coherent with 


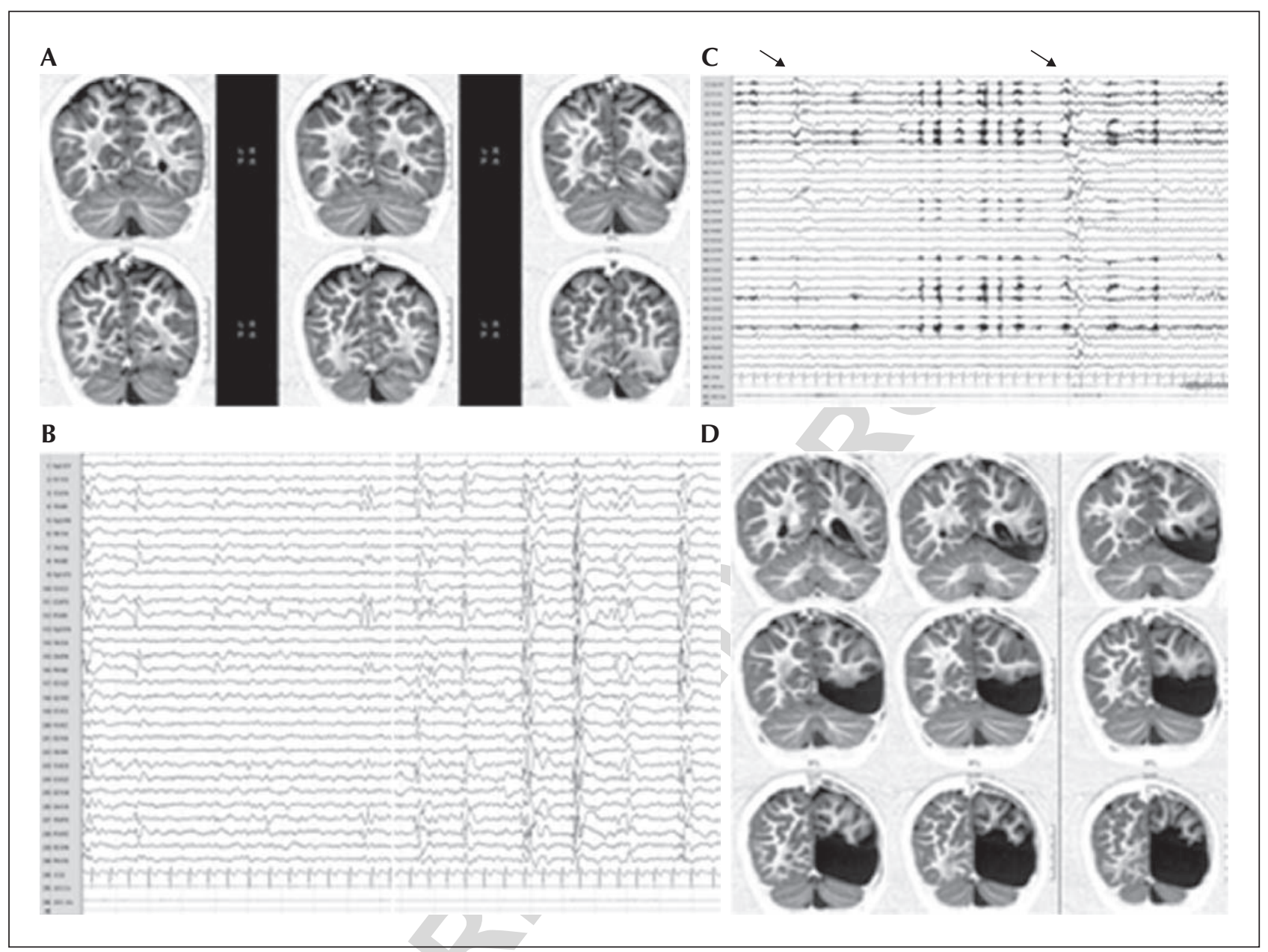

Figure 1. Patient 5 . Male, born $8 / 2003$, with no significant family or personal antecedents. Neurological examination revealed no focal deficits, the presence of slight convergent strabismus of the right eye, and moderate global deficit regarding psychomotor development. Epilepsy onset at 17 months of age with infantile spasms was preceded by an ocular rotation or deviation to the right. MRI showed a signal alteration involving the left occipital lobe, especially in the mesial part of the occipito-temporal junction (a). Interictal EEG showed slow waves and spike-waves on the left posterior region (b) with rapid diffusion to the anterior ipsilateral and posterior contralateral regions. Ictal EEG showed a bi-hemispheric rapid activity during spasms, slightly more rapid over the left occipito-parietal region (c). At 4 years of age, a tailored resection including the occipital mesial region (d) led to complete seizure freedom, causing a right $\mathrm{HH}$, behaviourally demonstrated since a formal visual field examination was not possible.

Histopathological analysis revealed FCD IIA. Neuropsychological evaluation at last follow-up visit showed a positive evolution of psychomotor development.

the epileptogenic side. The interictal EEG was correctly localising in 22 cases (35.5\%) and falsely/non-localising (bi-hemispheric or anterior localisation of interictal paroxysms) in 12 cases (19.3\%), while the distribution of interictal abnormalities in 25 cases $(40.3 \%)$ was within the posterior region. Ictal EEG was clearly localising in 21 cases $(37.5 \%)$, regional in $18(32 \%)$, and falsely/non-localising in 14 (25\%).

For 24 patients $(38.7 \%)$, a SEEG procedure was performed and in all cases was well tolerated; 272 spontaneous electroclinical events were recorded and a further 157 were elicited by cortical stimulation. For all subjects, SEEG evaluation provided clues that aided in the preservation of eloquent cortices and allowed to localise more precisely the EZ; in particular, interictal SEEG was localising in $46 \%$ ( 11 subjects), and ictal SEEG and stimulations were localising in $80 \%$ (19 subjects) and $67 \%$ (16 subjects), respectively.

\section{Imaging and histopathological features}

MRI demonstrated the presence of a discrete focal lesion in 58 cases $(93.5 \%)$, possible lobar abnormalities in 1 case, and was negative in 3. An MRI-visible lesion was sublobar in 12 cases (6 occipital: 4 mesial, 1 basal, and 1 lateral; 6 parietal: 3 mesial, 2 lateral, and 
A. Liava, et al.

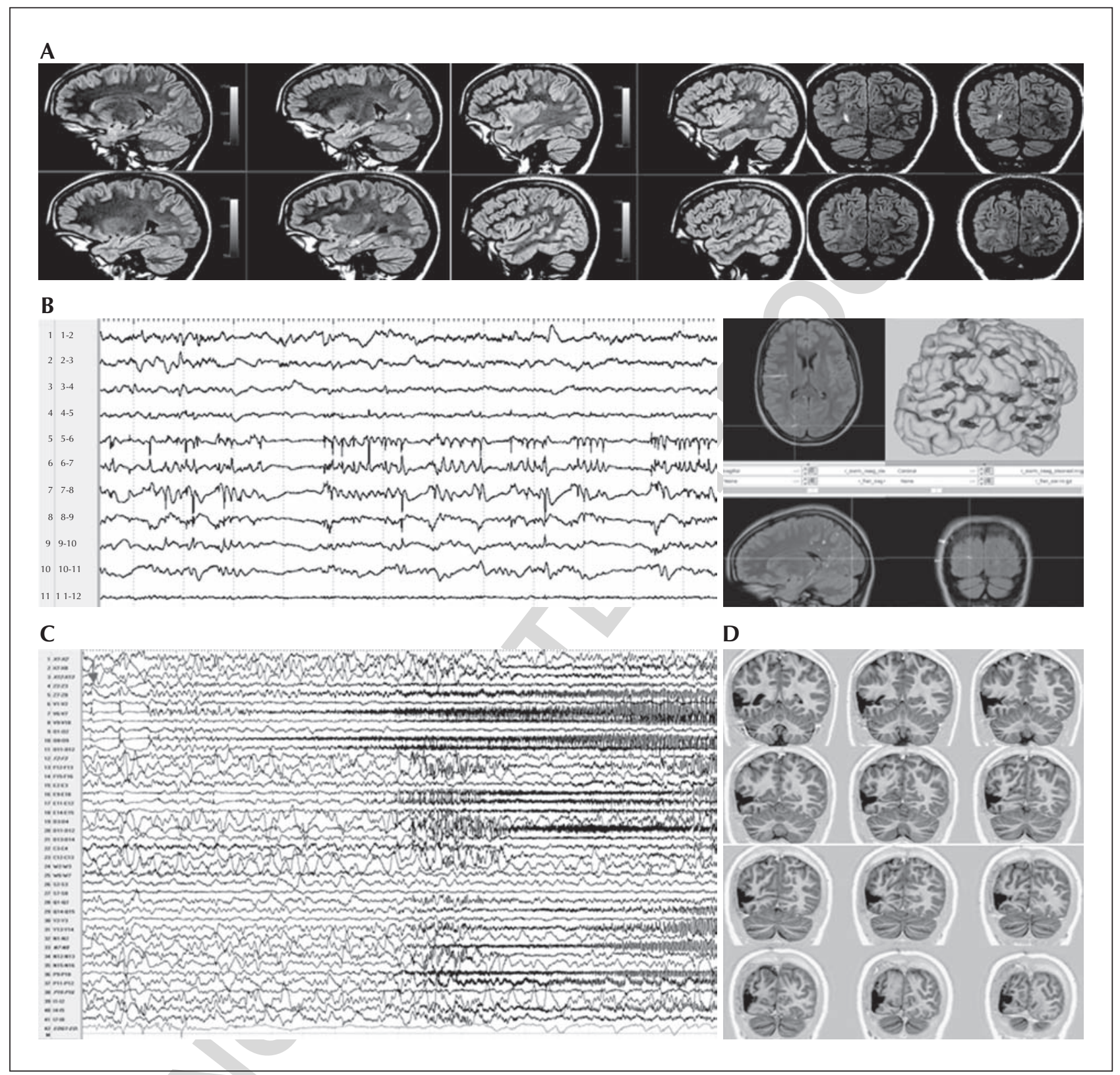

Figure 2. Patient 13. Female, born 9/1994, with no family or personal antecedents. The patient had normal neurological and neuropsychological examination, and was right-handed. Epilepsy onset at 5 years of age with weekly seizures was characterised by amaurosis and visual illusions without lateralisation, and followed by intense headache and, frequently, vomiting. This semiology resulted in a diagnosis and treatment of migraine for nearly two years, and afterwards, the ictal episodes were enriched with an initial saccadic deviation of the eyes to the left and blinking.

Interictal EEG showed the presence of spikes and spike-waves over the right PTO region and ictal EEG showed a right posterior ictal onset, while MRI revealed the presence of three distinct signal alterations, suggesting dysplastic lesions, localised over the right occipital pole, the OT junction at the level of the O2-O3 (and T2-T3) sulcus, and the intraparietal sulcus (a). An invasive evaluation with depth electrodes was performed in order to explore these malformative lesions, as well as the calcarine area, the posterior part of the hippocampus, the superior temporal gyrus, the parietal operculum, the hand area on the superior parietal region, and the inferior parietal lobule.

Interictal SEEG showed a sub-continuous spike activity intermingled with low-voltage fast discharges in the intermediate occipital contacts (see $[b]$, where all the traces derived from the electrode $\mathrm{V}$ are displayed).

Ictal SEEG (one of the 13 spontaneous seizures recorded during invasive monitoring is presented in [c]) revealed the presence of an intermediate occipital and dorso-lateral occipito-temporal EZ, the tailored resection of which (d) at 16 years of age, led to complete seizure freedom (at two years follow-up) without post-surgical visual field deficits.

Histopathological analysis revealed FCD II. 
1 opercular), lobar in 5 cases, involved two lobes in 25 cases, and was multilobar in the remaining 16 cases. Among the latter, MRI findings were compatible with hemi-hemimegalencephaly in 7 patients and ulegyria in 4 subjects.

Histopathological analysis confirmed FCD in 31 subjects (50\%; nine with FCD I and 22 with FCD II), tumoural lesions in 15 subjects (24\%; 6 with DNT, 6 with gangliogliomas, and 3 with other tumours), gliosis in $9(14.5 \%)$, cortical tubers in 3, and Sturge Weber in 2, while one subject presented with micropolygyria associated with nodular heterotopia, and no pathological abnormalities were identified in the excised tissue in another. Finally, hippocampal sclerosis was found in 5 cases.

\section{Surgical outcome}

A tailored resection was performed in the left hemisphere in 33 cases. Surgery consisted of 12 lesionectomies (Lt), 4 corticectomies $(\mathrm{Ct})$, and 6 functional disconnections (Dt), while 37 patients underwent $\mathrm{Lt}$ together with $\mathrm{Ct}$, and 3 subjects underwent Lt together with $\mathrm{Ct}$ and $\mathrm{Dt}$.

Permanent post-operative de novo or aggravated visual field deficits were observed in $32 \%$ of patients: 12 cases of $\mathrm{HH}$ and 8 cases of $\mathrm{HQ}$, all pre-operatively predicted and discussed with the families. Transient post-operative morbidity was reported in 2 children with a tailored resection in the $\mathrm{PL}$, involving a focal motor-sensitive deficit contralateral to the side of the excision.

Post-operative follow-up varied from 24 months to 16 years (mean: 6.92 years). Seizure freedom was achieved for $85.5 \%$ of subjects (53/62), in one case after a twostep operation; $77 \%$ (41/53) discontinued antiepileptic drugs. Among children who required a SEEG procedure, $75 \%$ achieved seizure freedom. The 3 patients with negative MRI achieved an unsatisfactory outcome (Engel class IIIa and IVa). In 5 cases, a partial resection of the MRI-identifiable lesion was performed; 4 subjects were cured and one was classified as class IVa. In 4 further cases, the resection of the EZ was incomplete, due to its functional intersection with eloquent sensorimotor areas; 2 patients were classified as class IIa and 2 as class IIIa. Thus, at the last follow-up visit, 53 patients were classified as Engel class I (52 as class Ia and 1 as class Id), 2 as class IIa, 4 as class IIIa, and 3 as class IVa.

\section{Groups as defined by the topography of resection}

Mean age at epilepsy onset was higher among the pure-OLE subjects, at 6 years, with respect to 3.27 years in the $\mathrm{O}+$ group, 3.2 years in the pure-PLE, and 1.45 years in the extended-OTP group.

The pure-OLE group presented with more preoperative visual field deficits; $62.5 \%$, compared to $29.4 \%$ in the extended-OTP, $19.2 \%$ in the O+ group, and $9 \%$ in the pure-PLE. However, in the pure-OLE group, visual field impairment was mostly not clinically significant (HQ). Children with pure-OLE exhibited more frequent visual aura (100\%; O+: $55 \%$; extended-OTP: 62.5\%; pure-PLE: none), positive EVH (83.3\%; O+: 31.2\%; extended-OTP: $10 \%)$, lateralised EVH (80\%; O+: $18.7 \%$; extended-OTP: none), ictal oculogyric movements ( $40 \%$; O+: $4.2 \%$; extended-OTP: $11.8 \%$; pure-PLE: none), epileptic nystagmus ( $40 \%$; $\mathrm{O}+: 20.8 \%$; extendedOTP: $23.5 \%$; pure-PLE: none), as well as a bi-occipital seizure onset on EEG ( $40 \%$; O+: $8.3 \%$; extended-OTP: $5.9 \%$; pure-PLE: none).

The extended-OTP group exhibited more frequent significant personal antecedents (29.4\%; pure-PLE: 27.3\%; pure-OLE: $12.5 \%$; O+: $7.7 \%$ ), neuropsychological impairment (64.7\%; pure-PLE: $45.5 \%$; O+: $38.5 \%$; pure-OLE: $37.5 \%)$, oculomotor deficits $(52.9 \%$; pureOLE: $25 \%$; O+: $11.5 \%$; pure-PLE: none), as well as a more marked pre-operative impairment of the visual field $(\mathrm{HH})(29.4 \%$; pure-OLE: $12.5 \%$; O+: $7.7 \%$; purePLE: none). Furthermore, spasms represented the most frequent seizure type in this group (47\%; O+: $19.2 \%$; pure-PLE: $18.2 \%$; pure-OLE: $12.5 \%$ ) and status epilepticus represented a frequent complication $(35.3 \%$; O+: 3.8\%; pure-OLE and pure-PLE: none).

Concerning the pure-PLE group, a neurological impairment, frequently in the form of spastic hemiparesis, was exhibited by a large proportion of children (45.5\%; extended-OTP: 17.6\%; O+: $7.7 \%$; pure-OLE: none),

\section{Figure 2. (Continued)}

\section{SEEG scheme:}

Electrode C: intermediate hippocampus, T1-T2 sulcus, middle temporal gyrus; Electrode D: para-hippocampal gyrus, fusiform gyrus, inferior temporal gyrus; Electrode E: anterior lingual gyrus, T2-T3 sulcus, posterior part of middle temporal gyrus; Electrode F: antero-inferior precuneus, middle temporal gyrus; Electrode I: antero-superior precuneus, superior parietal lobule; Electrode $\mathrm{N}$ : antero-superior precuneus, intraparietal sulcus, inferior parietal lobule; Electrode O: inferior calcarine cortex, middle occipital gyrus; Electrode P: splenium, intraparietal sulcus, superior parietal lobule; Electrode Q: parietal cingulate gyrus, angular gyrus; Electrode S: posterior insula, parietal operculum; Electrode V: superior calcarine cortex, superior occipital gyrus; Electrode W: postero-inferior insula, superior temporal gyrus; Electrode X: intermediate precuneus, intraparietal sulcus, superior occipital gyrus; Electrode Y: parietal cingulate gyrus, supramarginalis gyrus; Electrode Z: anterior cuneus, superior occipital gyrus. 
while, similarly, a large proportion presented with SGS (54.5\%; extended-OTP: 6\%; O+: 4\%; pure-OLE: none), more commonly within the first year following epilepsy onset. Moreover, hypertonic/dystonic or clonic contralateral ictal signs, as well as motor complex automatisms, were seen more frequently among the pure-PLE group ( $70 \%$ and $30 \%$, respectively).

Invasive recordings were performed in $54.5 \%$ of the pure-PLE group, $46.1 \%$ of the O+ group, and $35.3 \%$ of the extended-OTP group, whereas invasive evaluation was obviated for all patients of the pure-OLE group. Seizure freedom was achieved for all patients of the pure-OLE group, in $92.3 \%$ of the $\mathrm{O}+, 82 \%$ of the purePLE, and $70.6 \%$ of the extended-OTP group.

Tumours represented the most common histopathological abnormality among the pure-OLE group $(62.5 \%)$ and gliotic lesions were found more frequently among the extended-OTP subjects $(29.5 \%)$, while dysplastic lesions were observed most frequently and almost equally in both the $\mathrm{O}+$ and the extended-OTP group $(61.5 \%$ and $65 \%$, respectively). In the pure-PLE group, tumoural lesions were found in $36.3 \%$ and malformations of cortical development in $54.5 \%$ of cases.

\section{Oculomotor manifestations}

In 9 cases, a clonic eye deviation was associated with a "clonic" (rhythmic spiking or spike-wave) ictal discharge on EEG/SEEG, involving the contralateral occipital region, while a tonic contralateral eye deviation was always associated with a "tonic" (low-voltage fast activity) discharge. Moreover, when a tonic ictal discharge became progressively slower and rhythmic, the sustained eye deviation became saccadic, with the eyes moving horizontally and not crossing the midline, until the end of the ictal oculomotor event. Among the patients studied by SEEG, high-frequency electrical stimulations $(50 \mathrm{~Hz}, 1$ msecond width, $1-3 \mathrm{~mA}$, during 2-6 seconds) elicited the following ocular movements: - a contralateral tonic eye deviation was provoked by stimulating the posterior lingual gyrus in 3 cases, the anterior cuneus in 3 cases, the intermediate occipital gyrus in 3 cases, and the fusiform gyrus and the intraparietal sulcus each in 1 case;

- an ipsilateral tonic eye deviation was observed when stimulating the posterior para-hippocampic gyrus and the anterior cuneus;

- a monocular sustained deviation consisting of a convergent strabismus was seen when stimulating the contralateral anterior cuneus and associated with a sensation of monocular movement when stimulating the contralateral lingual gyrus;

- oculogyric movements were provoked by stimulating the intermediate occipital gyrus and associated with a sensation of eye movement when stimulating the anterior part of the lateral occipito-temporal sulcus.

We did not consider eye deviations associated with visual hallucinations since an eye version could have been the result of gaze orientation towards the hallucination.

\section{Prognostic elements}

The following factors were identified to correlate with surgical outcome (table 3): (i) younger age at epilepsy onset correlated with outcome in a negative way; all but one subject with seizure onset after the first year achieved seizure freedom, with respect to $71.4 \%$ of those with epilepsy onset in the first year of life $(p=0.00521)$; (ii) the presence of a neuroradiologically-identifiable lesion represented a favourable prognostic factor $(p=0.00798$ ); and (iii) a complete excision of the EZ represented a strong positive predictive element $(p=0.00148)$, whereas a complete resection of the neuroradiologically-visible lesion did not correlate with outcome $(p=0.31953)$. However, after applying the correction for multiple comparisons, none of these features maintained a statistically significant predictive value.

Factors unrelated to surgical outcome were: epilepsy duration, seizure frequency, the presence of aura, localising interictal and ictal EEG findings, pathology, necessity of invasive evaluation, and topography of resection.

\section{Discussion}

To our knowledge, this is the largest series of an exclusively paediatric patient population who underwent tailored surgery for pharmaco-resistant PCE, with at least two years of post-operative follow-up (mean follow-up: 6.92 years). Despite the fact that the sensitivity of MRI in this series (93.5\% MRI-positive) is comparable to previous series (Binder et al., 2008; Binder et al., 2009; Tandon et al., 2009), the excellent post-operative seizure outcome is more encouraging relative to previous reports (Binder et al., 2008; Binder et al., 2009; Tandon et al., 2009; Jobst et al., 2010; Ibrahim et al., 2012).

As a whole, the general characteristics of our population did not differ from those in other surgical series; the majority of the children had a normal neurological examination, moderate neuropsychological impairment, and a frequent pre-operative visual field defect, as well as an oculomotor deficit. Furthermore, they mostly exhibited a severe epilepsy with high seizure frequency, an early epilepsy onset frequently associated with infantile spasms, complex seizure patterns resulting from the rapid propagation of ictal 
Table 3. Prognostic elements (two-tailed Fisher exact test and student t-test).

\begin{tabular}{|c|c|c|c|c|c|}
\hline Variable & Total & Class I & Non-Class I & $P$ value & $\begin{array}{l}\text { Bonferroni } \\
\text { correction }\end{array}$ \\
\hline Epilepsy duration & $\begin{array}{l}\text { mean: } \\
5.5 \text { years }\end{array}$ & $\begin{array}{c}\text { mean: } 5.52 \text { years, } \\
\text { DS: } 4.29\end{array}$ & $\begin{array}{c}\text { mean: } 6 \text { years, } \\
\text { DS: } 4.89\end{array}$ & NS & \\
\hline \multicolumn{6}{|c|}{ Number of patients } \\
\hline \multicolumn{6}{|l|}{ Age at onset } \\
\hline over 1 year & 34 & 33 & 1 & 0.00521 & 0.06773 \\
\hline under 1 year & 28 & 20 & 8 & & \\
\hline \multicolumn{6}{|l|}{ Seizures frequency } \\
\hline daily & 41 & 34 & 7 & 0.23273 & \\
\hline non-daily & 21 & 19 & 2 & & \\
\hline Aura & 36 & 34 & 3 & 0.07558 & \\
\hline No aura & 26 & 20 & 6 & & \\
\hline No SEEG & 38 & 35 & 3 & 0.05597 & \\
\hline SEEG & 24 & 18 & 6 & & \\
\hline MRI+ & 58 & 52 & 6 & 0.00798 & \\
\hline MRI- & 4 & 1 & 3 & & \\
\hline Tumours/DNTs & 15 & 15 & 0 & 0.07861 & \\
\hline FCD & 31 & 25 & 6 & & \\
\hline FCD & 31 & 25 & 6 & 0.29628 & \\
\hline Other histology (except tumours) & 15 & 13 & 2 & & \\
\hline \multicolumn{6}{|l|}{ MRI-visible lesion } \\
\hline Incomplete resection & 5 & 4 & 1 & 0.31953 & \\
\hline Complete resection & 53 & 49 & 4 & & \\
\hline \multicolumn{6}{|l|}{ EZ } \\
\hline Incomplete resection & 3 & 0 & 3 & 0.00148 & 0.01924 \\
\hline Complete resection & 59 & 54 & 5 & & \\
\hline \multicolumn{6}{|l|}{ Resection topography } \\
\hline Extended-OTP & 17 & 12 & 5 & 0.04545 & \\
\hline Remaining groups & 45 & 41 & 4 & & \\
\hline \multicolumn{6}{|l|}{ Interictal EEG } \\
\hline Localising & 22 & 21 & 1 & 0.0834 & \\
\hline Non-localising & 40 & 32 & 8 & & \\
\hline \multicolumn{6}{|l|}{ Ictal EEG } \\
\hline Localising & 21 & 19 & 3 & 0.30255 & \\
\hline Non-localising & 35 & 30 & 5 & & \\
\hline
\end{tabular}

NS: non significant; EZ: epileptogenic zone.

discharges, and a high incidence of dysplastic lesions that rarely appeared restricted. Increased awareness of the clinical and morpho-functional peculiarities, indicating a posterior location of the seizure focus, resulted in an improvement in the rate of surgical success in the present series.

\section{Ictal semiology}

The diversity of ictal manifestations in parietal lobe origin seizures in our series, such as focal tonic seizure activity and seizures with automatisms, confirm once more the misleading semiology of PL seizures (Blume 
et al., 1991 Q4; Salanova et al., 1993 Q5; Ristic et al., 2012) which reflects the rapid seizure spread outside of the PL either to the frontal region, most commonly the supplementary motor area, or to the temporolimbic structures. Furthermore, a large proportion of the pure-PLE patients exhibited SGS, most commonly within the first year following epilepsy onset. This feature is in line with previous series (65-84.2\%; Kim et al. [2004a], Kim et al. [2004b], Binder et al. [2009]), suggesting that secondary generalisation is more frequent in PLE than in other epilepsies.

With respect to the patients who benefited from an excision involving the $\mathrm{OL}$, our findings are concordant with the existing literature in which the prevalent clinical pattern of seizures, confirmed to be of occipital origin, is temporal (Salanova et al., 1992; Williamson et al., 1992; Blume et al., 2005; Binder et al., 2008; Tandon et al., 2009; Jobst et al., 2010) and less frequently frontal (Ludwig and Marsan, 1975). Half of the children (25/51) in our series exhibited a temporal seizure pattern, mostly characterised by autonomic/vegetative manifestations and oro-alimentary automatisms, whereas $35.3 \%$ (18/51) presented with a frontal pattern, mainly consisting of an axial deviation of the head followed either by a hemiclonic or an aversive seizure.

\section{Subjective ictal manifestations}

There is general agreement concerning the high $\mathrm{OL}$ localising value of early visual lateralised simple hallucinations (Penfield and Jasper, 1954; Ludwig and Marsan, 1975; Salanova et al., 1992; Williamson et al., 1992). In our series, $63.6 \%$ of the elementary visual aura was reliably lateralising, being contralateral to the epileptogenic side; lateralised EVH were exhibited mainly by the group presenting with a pure occipital ictal onset (pure-OLE: $80 \%$; O+: 18.75\%; extended-OTP and pure-PLE: none). Furthermore, with respect to cortical stimulations performed in our patients, lateralised $\mathrm{EVH}$ were described when the discharges were well localised in the peri-calcarinian areas. Stimulation of the mesial occipital region above the calcarine fissure induced phosphenes in the lower visual field, contralateral to the stimulated hemisphere, while stimulation below the calcarine fissure produced phosphenes in the contralateral upper visual field, in concordance with previous reports (Munari et al., 1993; Lee et al., 2000).

None of our pure-OLE patients presented $\mathrm{CVH}$ $\mathrm{Vl}$; these symptoms were reported mainly by the children who benefited from occipito-temporal or occipito-parietal resections, apparently in relation to the high-order visual processing areas located in that region. In fact, $\mathrm{CVH}$ appear if the basal temporooccipital cortex is stimulated (Bien et al., 2000; Lee et al., 2000; Jobst et al., 2010), usually in the fusiform gyrus and the lateral temporal or temporo-occipital cortex. Additionally, VI can be elicited by stimulation of the basal temporo-occipital cortex and the inferior parietal lobule (Penfield and Jasper, 1954).

Ictal blindness was described almost equally in the three groups who benefited from a resection involving the OL. It has been reported that amaurosis or large visual-field defects are induced by the stimulation of the lateral temporo-occipital and temporo-parieto-occipital junctional regions, even if the pathophysiology of this symptom remains rather unclear (Lee et al., 2000).

Beyond visual aura, fear was the most frequent ictal subjective symptom, probably reflecting the child's reaction to the perception of seizure onset rather than an ictal emotional change (Liava et al., 2012). Interestingly, 6 of the 7 patients reporting this symptom benefited from a resection involving the parietal lobe. Fear and anxiety were previously described as a manifestation of parietal seizures (Alemayehu et al., 1995), reaching an incidence of $17 \%$ (Bartolomei et al., 2011), while previous studies have reported the presence of deficits in recognition of fearful faces in patients with parietal lesions (Adolphs, 2002).

Even if the small patient number is taken into account, $50 \%$ of children with aura, who benefited from a purePLE resection, reported contralateral paresthesias and vertigo, which are the most common subjective manifestations of PL seizures (Salanova et al., 1995; Kim et al., 2004a; Kim et al., 2004b; Bartolomei et al., 2011); the latter symptom has been attributed to seizure activity involving the vestibular cortex (Kahane et al., 2003) in the temporal-parietal junction or involving the superior parietal region (Bartolomei et al., 2011), while vertiginous sensations have been induced by cortical stimulation in the intraparietal sulcus (Dieterich, 2007).

\section{Objective ictal manifestations}

The presence of ictal opsoclonic/oculogyric movements represents an interesting feature of occipital seizures. These oculomotor manifestations are not frequent; they are discrete and recognition is dependent on careful observation. In our series, this sign was present in 5 children (11\%), in all cases as a leading ictal sign, in line with previous reports (Fogarasi et al., 2003). Oculogyric movements were observed mainly in the pure-OLE group and in 2 cases this manifesta- 
tion was associated with a sensation of eye movement. Previous contributions have reported a sensation of "eye pulling" in association with occipital seizures (Williamson et al., 1992), however, the neurological underpinnings of this symptom are incompletely explained at present (Tandon et al., 2009). We elicited this kind of subjective manifestation in 2 patients by stimulating the anterior part of the lateral occipitotemporal sulcus and the lingual gyrus. Previous studies have reported that moving sensations were evoked by stimulation of the basal temporo-occipital and the mesial parieto-occipital or temporo-parieto-occipital junctional regions (Lee et al., 2000).

Epileptic nystagmus, defined as quick repetitive eye movement secondary to epileptic activity, has been reported in about $0.5 \%$ of patients in the epilepsy monitoring unit and is noted predominantly in children (Kellinghaus et al., 2008). In our series, epileptic nystagmus was exhibited by 11 children (24\%); it had a lateralising value, always occurring contralateral to the epileptogenic side and was more frequent in the pure-OLE group. Epileptic nystagmus is reported to have limited localising value, presumably due to inconsistent data with regards to the pathophysiological mechanisms by which it is generated (Takeda et al., 1970; Godoy et al., 1990; Weber et al., 2006 Q6; Kellinghaus et al., 2008). Our results are in concordance with Fogarasi et al. who found that $39 \%-47 \%$ of children under 12 years of age with symptomatic posterior cortex epilepsy presented with ictal nystagmus (Fogarasi et al., 2003; Fogarasi et al., 2005).

Oculomotor manifestations, consisting mostly of contralateral tonic eye deviation, represented the leading ictal sign for a large proportion of children $(85 \%)$. In contrast, the localising value of early eye deviation has been considered differently (Munari et al., 1993). Munari et al. (1984) evaluated 49 occipital seizures, with an early ocular deviation recorded during SEEG, and found that eye deviation was related to the ictal disorganisation of the mesial occipital cortex, was always contralateral, was not related to extra-occipital discharges, and was, more frequently, of a tonic type (44/49). Furthermore, Takeda et al. (1970), in a study of 250 occipital seizures, concluded that eye-head tonic deviation, as well as oculo-clonic eye movements, occur in relation to the primary involvement of the occipital lobe and constitute the most frequent leading ictal sign. This data appears to be in to contrast to other studies (Penfield and Jasper, 1954; Godoy et al., 1990) showing that contralateral eye deviation is induced by frontal eye field (FEF) stimulation. Nonetheless, recent data, mainly derived from $\mathrm{fMRI}$ and TMS studies (Pierrot-Deseilligny et al., 2004), show that pursuit eye movements are linked not only to the activity of the lateral intra-parietal area, which is specialised for saccadic eye movements and strongly connected with the FEF, but also to the activity of a second eye field in the medial parietal area, located in the proximity of the angular gyrus, the electrical stimulation of which also provokes eye movements (Thier and Andersen, 1998). In our series, in the few cases in which an isolated eye deviation was evoked by electrical stimulation, the involved structures included the intermediate occipital gyrus, the anterior cuneus, and the lingual gyrus.

In fact, in order to ascribe a clear localising value to eye deviation, it is mandatory to evaluate this sign in the context of the global semiological picture of each patient; some associations with ictal signs and symptoms appear to provide a higher localising value. For instance, an eye/eye-head deviation associated with a tonic manifestation of the limbs could evoke a frontal dorsolateral ictal onset (Manford et al., 1996); a head version following an eye deviation and associated with complex postural manifestations might suggest a SMA ictal disorganisation (Bleasel and Dinner, 2008); an eye deviation followed by head orientation, and not associated with other somatomotor components, might suggest the presence of visual hallucinations and the initial ictal perturbation of the occipital cortex, while a slow eye deviation associated with oculoclonic manifestations may indicate an occipital origin.

\section{EEG features}

The poor lateralising and localising value of scalp EEG, reported in previous studies (Jobst et al., 2010), has been related to the rapid spread of the OL-originating seizures to the neighbouring regions (Blume et al., 2005) as well as to the fact that most of the OL is buried, making the identification and precise localisation of interictal discharges challenging (Tandon et al., 2009). Similarly, several reports have emphasized that surface EEG monitoring is often non-localising in PLE (Williamson et al., 1992; Cascino et al., Q7; Kim et al., 2004 Q8). However, in our series, interictal EEG was misleading in only $19.3 \%$ of cases because of frontal or generalised interictal discharges, while in the remaining cases, a posterior predominance of the interictal discharges was observed. Ictal EEG similarly showed a posterior ictal onset in $69.6 \%$ of cases, with either a subsequent early spread to the frontal regions or a rapid involvement of the contralateral posterior region.

In this regard, a posterior temporal interictal focus on scalp EEG, a relatively frequent finding in OLE (Salanova et al., 1992; Palmini et al., 1993), does not necessarily have a negative prognostic value, as previously reported (Jehi et al., 2009), but might serve as a marker for a possible occipital EZ when other signs and 
symptoms point to the occipital region (Williamson et al., 1992). Indeed, temporal spiking is associated with the abundant projections from the OL to the lateral and mesial temporal structures. This EEG feature, therefore, in the absence of temporal abnormalities based on imaging or very early semiology implying involvement of temporal areas (abdominal, psychic, or complex visual aura), cannot be considered as a negative prognostic feature.

Globally, our results suggest that EEG features are important for providing an indication of "posterior localisation", but are not accurate enough to precisely define the "what-to-remove area" (Kahane et al., 2006). In this view, interictal and ictal scalp EEG were poorly localising ( 35.5 and $37.5 \%$ of cases, respectively) due to poor spatial resolution.

\section{Resection topography}

The extension of the resection was always individually tailored on the basis of each patient's anatomo-electroclinical characteristics. Nonetheless, four main groups of patients were identified. The pure-OLE group presented with a later epilepsy onset, exhibited a higher incidence of tumoural lesions, and obtained excellent post-operative results without invasive presurgical evaluation due to the good correlation between electro-clinical data and location of the lesion.

The $\mathrm{O}+$ group exhibited a slightly less favourable seizure outcome (92.3\% seizure-free) and frequently required invasive recordings $(46 \%)$. The majority of the underlying lesions responsible for an O+ epilepsy were represented by relatively "restricted" cortical dysplasias which involved the temporal or/and parietal cortex, adjacent to the OL. Indeed, FCD is rarely a localised process, but rather a functionally, electrophysiologically, and clinically integrated neural network disorder (Duchowny, 2009), exhibiting multifocal dysfunctional features and poorly delineated pathways which might be intersected with functionally eloquent areas. In this regard, individually-tailored surgery has more of a chance to be curative when guided by invasive investigation (figure 2). In fact, $89 \%$ (11/12) of the children with an O+ epileptic focus who underwent a SEEG evaluation achieved complete seizure freedom.

Similarly, a large proportion of the pure-PLE group required invasive evaluation $(54.5 \%)$, not only due to the diversity of ictal signs, but also in order to map eloquent cortex for sensorimotor function (figure 3), since functional imaging does not always provide adequate informative clinical value regarding the final surgical decision, especially in children in whom functional neuroradiological studies are difficult to perform.
The most common imaging abnormalities among children who benefited from more extended resections consisted of hemi-hemimegalencephaly and ulegyric lesions. Hemi-hemimegalencephaly or posterior quadrantic dysplasia is a rare malformation, involving the $\mathrm{OL}$ and the posterior part of parietal and temporal lobes. It is associated with early-onset pharmacoresistant epilepsy and immature seizure patterns such as spasms and, consequently, with a deterioration of psychomotor development (D'Agostino et al., 2004). Ulegyria is included among the hypoxic-ischaemic encephalopathies; it shows a peculiar MRI presentation and is frequently associated with perinatal complications, medically refractory epilepsy, and intellectual disability (Gil-Nagel et al., 2005). Given the severe epilepsy sustained by these conditions, and since patients with infantile epileptic encephalopathy may have a better post-surgical outcome, especially in terms of developmental gains, early surgery aiming to completely remove the abnormal tissue must be considered (figures 4 and 5). In our series, the presence of these neuroradiological findings associated with concordant EEG, clinical, and neuropsychological features, obviated the need for an invasive presurgical evaluation $(11 / 17 ; 64.7 \%)$ and nonetheless, resulted in a satisfactory outcome $(9 / 11 ; 82 \%$ Engel class la). However, the extended-OLE group had the overall worst outcome $(70.6 \%$ seizure-free), probably related to poor visualisation of the limits of widespread abnormal tissue based on radiology and consequently the difficulty in delineating the epileptogenic foci. Indeed, the presence of a rather restricted and well-circumscribed $\mathrm{EZ}$, the complete resection of which was possible to accomplish, was a factor suggestive of favourable surgical outcome.

\section{Post-operative visual deficit}

It emerges from most studies that visual field postsurgical deficits following PCE surgery occur in a high proportion of patients; preservation of visual function is likely to become more common with advancing structural and functional imaging (Tandon et al., 2009). However, despite the developments in functional neuroimaging, namely tractography, post-operative visual field deficits following PCE surgery are difficult to avoid and are still reported to be relatively high (38-81\%; Boesebeck et al., 2002; Lee et al., 2005 Q9; Binder et al., 2008; Tandon et al., 2009). In our series, $32 \%$ of patients experienced de novo or aggravated post-operative visual field impairment. Stimulation mapping of primary visual cortex and visual pathways represented a milestone for visual outcome, since imaging techniques, such as tractography, were not always available during the time period in which these patients were 
A

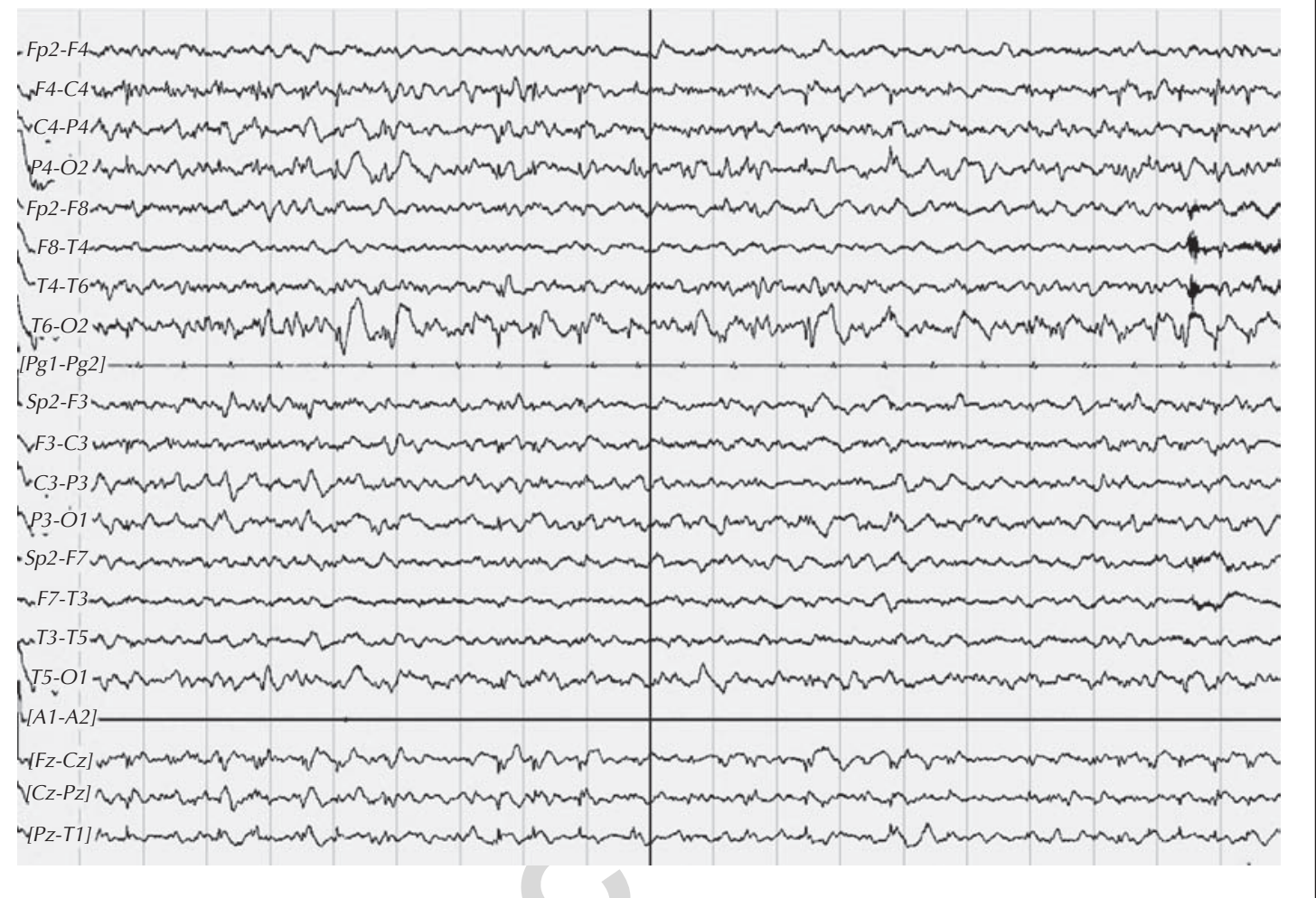

Figure 3. Patient 61. Male, born $1 / 2000$, with no significant family or personal antecedents. Neurological examination was normal. The patient was shown to have an inferior quadrant visual field defect, moderate neuropsychological deficits concerning discrimination of simple visual pattern, and reading abilities. Epilepsy onset occurred at 5 years of age with a secondary generalised seizure preceded by crying. Five days later, the child presented with focal seizures with a high frequency, characterised either by an isolated myoclonus of the left leg or, more commonly, by subjective symptoms consisting of a feeling of something "scary"; the child would cry and search for help, and afterwards, stare, with eyes wide open, mimicking fear. This was followed by agitation, crying, and repetitive and frantic movements with both arms and legs, over a duration of about 15-30 seconds, with no postictal deficits.

Interictal EEG showed spikes and sharp waves in the right temporal-parietal regions, intermixed with slow waves during slow sleep (a). Ictal VEEG showed the presence of seizures characterised by a dystonic posturing of the left arm associated with motor automatisms, correlated with a right temporal-parietal flattening, followed by slow monomorphic activity.

MRI showed a possible structural alteration of the right intra-parietal sulcus (b).

SEEG (c), performed at 6 years of age, revealed the presence of interictal paroxysms localised in the superior parietal lobule and in the intraparietal sulcus, up to the mesial portion of the cuneus ( $d$ and e), the presence of hypermotor seizures and seizures with hypertonic/dystonic posturing, and an epileptogenic area involving the precuneus and the paracentral lobule (f). The resection was confined to the mesial portion of the parietal lobe in order to avoid a left hemiparesis (g). The child was seizure-free for about three years; afterwards, weekly seizures re-appeared, constituting an "electric-like" sensation in the left leg associated with pain, which could sometimes cause a fall. Histopathological analysis revealed FCD I and gliosis.

\section{SEEG scheme:}

Electrode P: precuneus, intraparietal sulcus, inferior parietal lobule; Electrode N: precuneus, sulcus in the superior parietal lobule, superior parietal lobule, inferior parietal lobule; Electrode L: precuneus, intraparietal sulcus, inferior parietal lobule; Electrode Q: mesial portion of cuneus, middle temporal gyrus; Electrode V: calcarine cortex, middle occipital gyrus; Electrode E: posterior portion of lingual gyrus, inferior occipital gyrus; Electrode W: superior portion of lingual gyrus, superior portion of middle temporal gyrus; Electrode K: superior parietal lobule; Electrode J: portion of cingulum inferior to the paracentral lobule, inferior portion of central sulcus, post-central gyrus; Electrode $X$ : parietal cingulum, supramarginalis gyrus. 
A. Liava, et al.

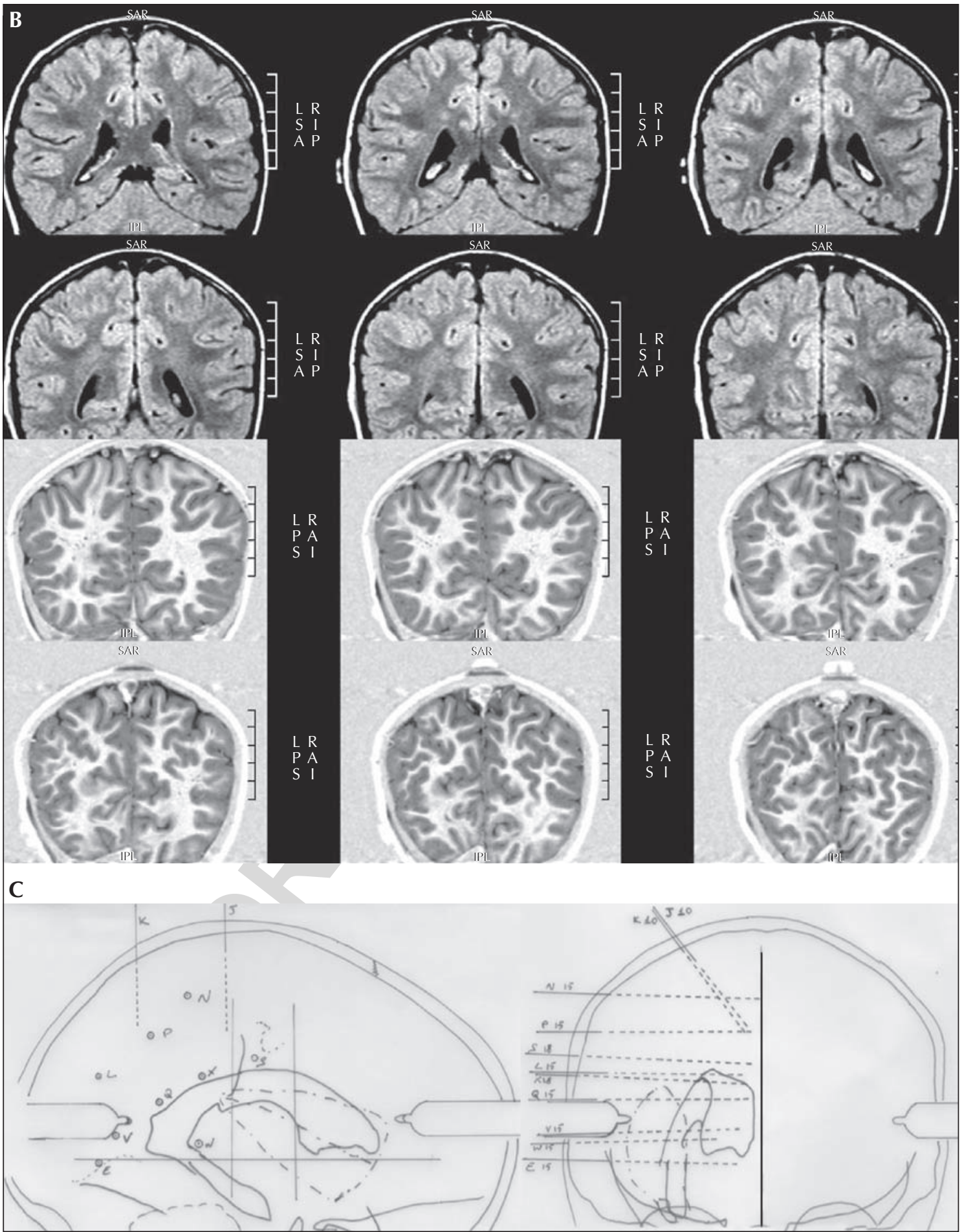

Figure 3. (Continued) 


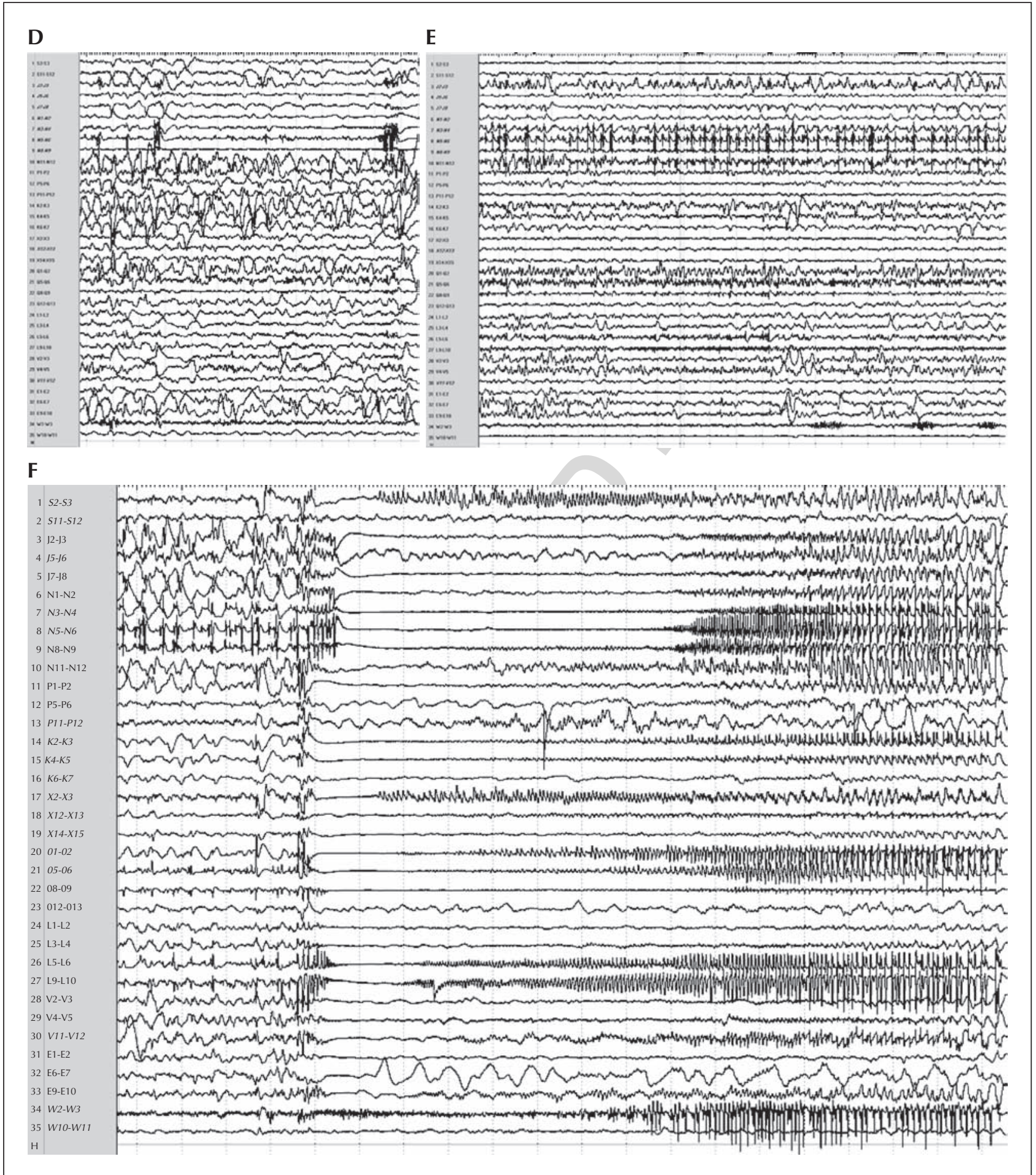

Figure 3. (Continued) 


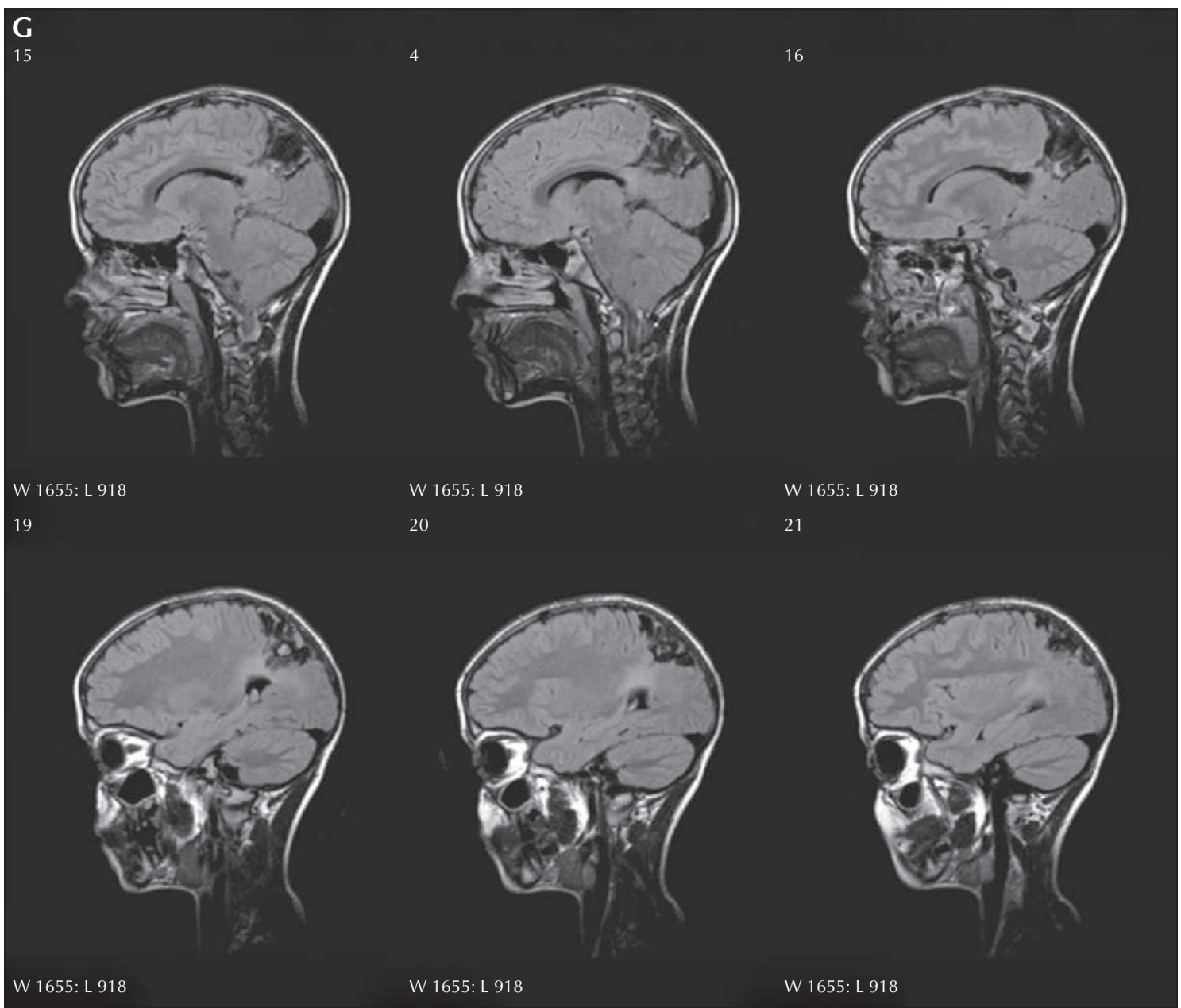

Figure 3. (Continued)

treated. Post-surgical $\mathrm{HH}$ was more frequent in the extended-OLE group, related to a more extended surgical excision. However, loss of visual function in the contralateral hemi-field in the context of a severe earlyonset epilepsy could be an acceptable trade-off to avoid epileptogenesis-related deterioration of higher cortical functions.

\section{Concluding remarks}

1. Epilepsy surgery can be a highly effective treatment for symptomatic PCE in paediatric age.

2. Accurate analysis of the chronology of ictal semiology and electrophysiological features, viewed in the context of the whole electroclinical pattern, provides a topographical orientation in PCE, the diagnostic complexity of which has been emphasized in the literature.
3. The presence of a pre-operative visual field deficit, frequently not clinically-significant, along with lateralised EVH and first ictal signs consisting of oculogyric and oculoclonic movements, are highly suggestive of an occipital EZ. These ictal signs are uncommon; they are discrete and recognition is dependant on careful observation.

4. Excellent results can be obtained in pure-OLE without invasive presurgical evaluation in carefully selected patients with a neuroradiologicallydemarcated lesion, of which the location correlates with electroclinical data. On the other hand, precise localisation in pure-PLE often requires invasive investigations, even in the presence of MRI-detectable lesions, given the misleading semiology and the necessity to map functional sites of sensorimotor function.

5. Posterior quadrantic dysplasia and ulegyria are frequent aetiologies of posterior cortex epilepsy in 


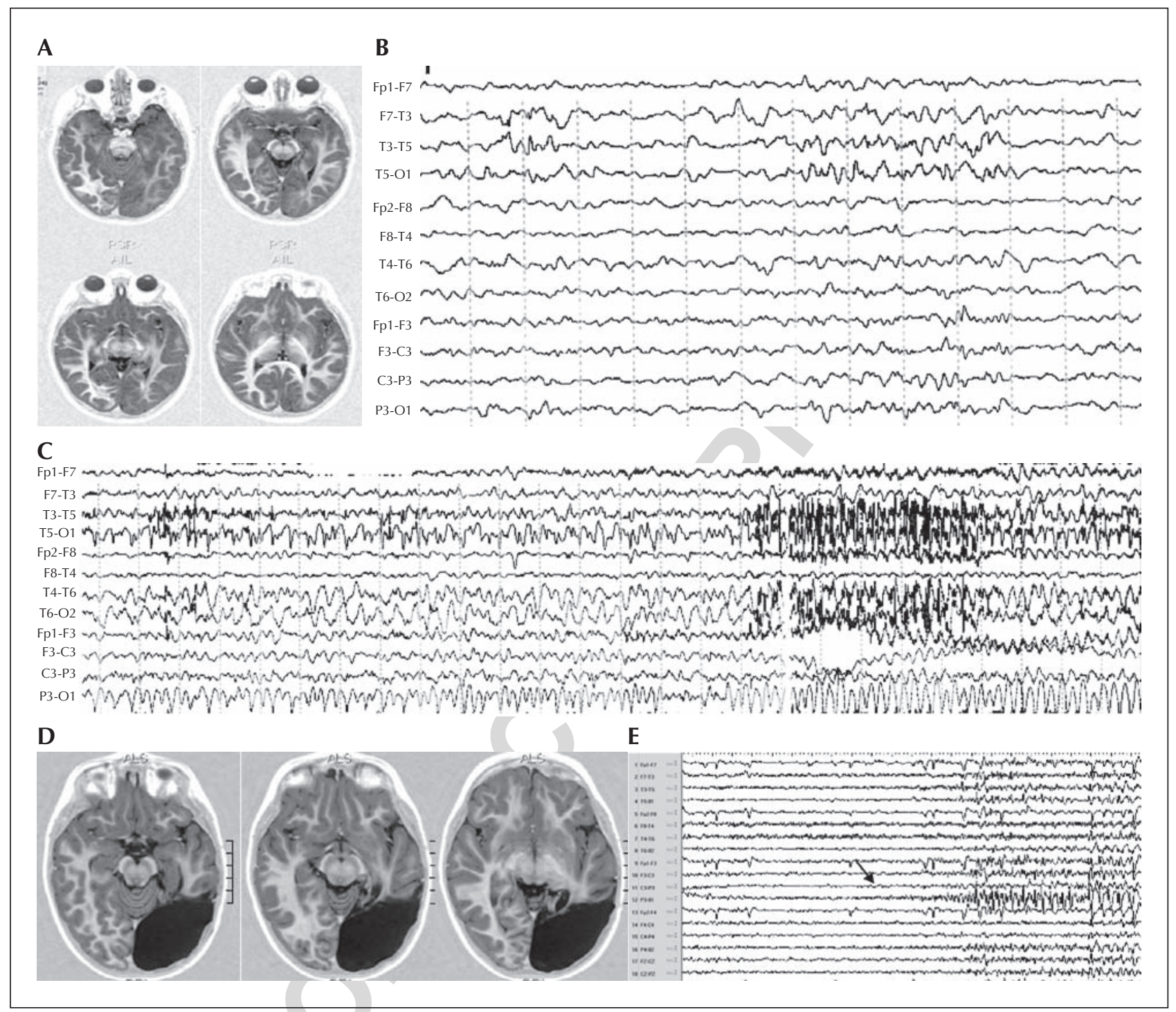

Figure 4. Patient 35. Male, born 1/2001, with no family or personal antecedents. Neurological examination revealed no focal deficits, mild psychomotor impairment, slight divergent strabismus of the right eye, and probable right hemianopic deficit. Epilepsy onset on the eighth day of life occurred with highly frequent seizures, characterised by horizontal nystagmus beating rapidly towards both sides, apparently without loss of contact. After several months, the ictal episodes constituted a sustained eye deviation to the right, followed by blinking and pendular horizontal nystagmus; sometimes the seizure continued with a postural hypertonia of the right limbs, while the child also presented with daily dialeptic seizures.

MRI revealed the presence of a vast TPO dysplastic lesion (a).

Interictal EEG showed the presence of left OT subcontinuous delta activity, intermixed with spikes and a short burst of fast activity, localised over the left OT region and diffused to the posterior temporal and, to a lesser degree, to the OP region (b), while ictal EEG showed a left $\mathrm{O}$ ictal onset (c). A partial lesionectomy consisting of a tailored OT resection (d) led to complete seizure freedom for nearly four years, associated with developmental gain. Afterwards, the seizures reappeared and were characterised by amaurosis, horizontal monocular nystagmus, head deviation to the right, blinking, lip corner deviation ("smile"), and hypertonia of the right limbs.

EEG showed rare spike-waves, localised over the left temporo-parietal region and a left parieto-occipital ictal onset with early temporal spread (e).

An SEEG investigation was performed in the left parieto-temporal region and showed: subcontinuous interictal parietal spikes (f; electrode Q7-8), an independent frequent infraclinical paroxysmal activity in the mesial temporal region (f; electrode C4-5), and an ictal onset over the intermediate mesial inferior parietal region ( $f$; electrode Q2-3). The resection was extended to the inferior parietal region, but a complete disconnection of the temporal lobe was also performed in order to achieve complete posterior quadrant surgery (g). Histopathological examination revealed FCD IIA. The child was classified as Engel class la since 2007 and AEDs were definitively stopped in 2012. The neuropsychological evaluation at five years follow-up demonstrated a normal psycho-cognitive level. 


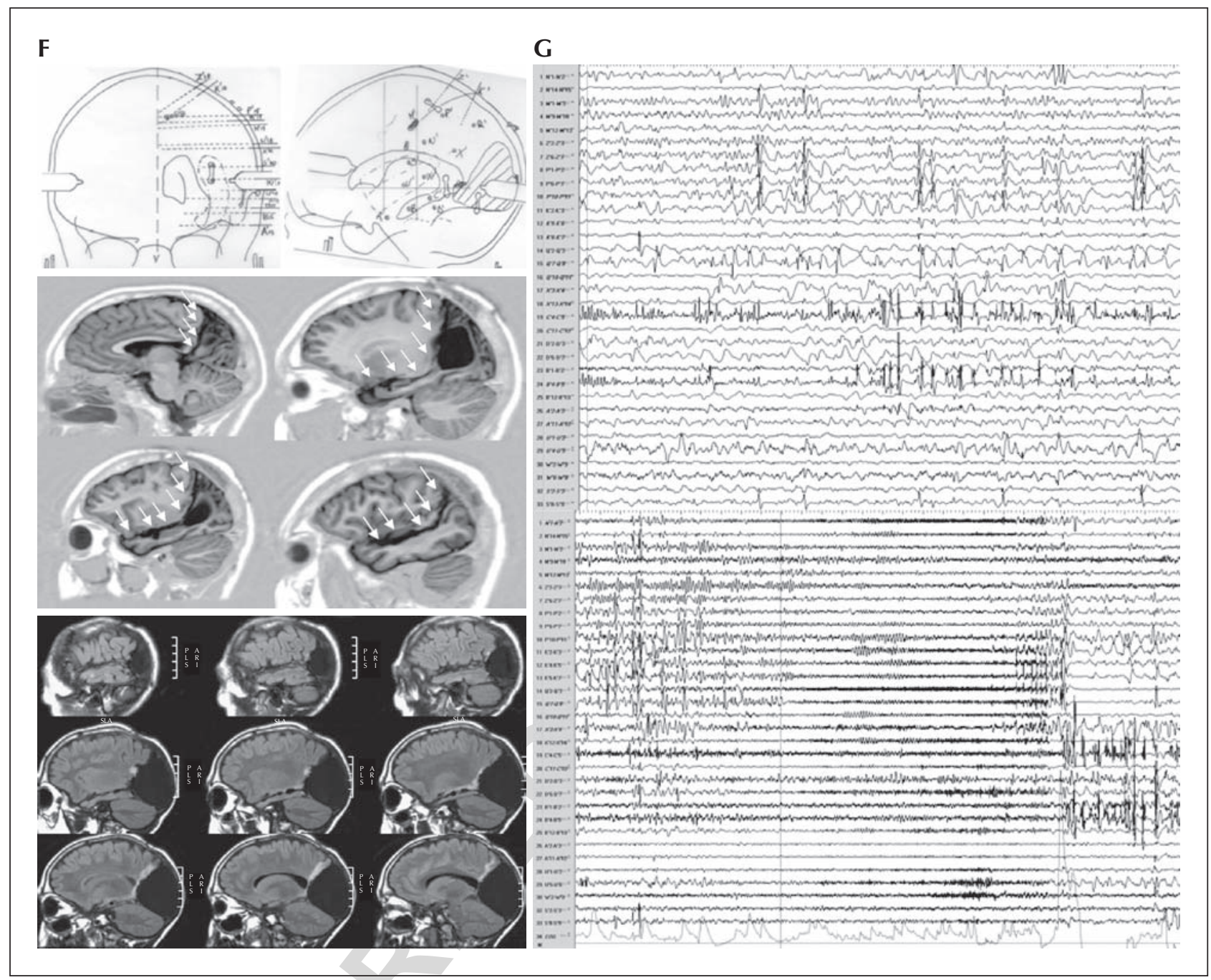

Figure 4. (Continued)

Scheme SEEG:

Electrode N: parietal cingulate gyrus, inferior postcentral gyrus; Electrode B: anterior parahippocampic gyrus, hippocampus, anterior fusiform gyrus, intermediate part of inferior temporal gyrus; Electrode Q: posterior precuneus, posterior part of superior parietal lobule, posterior part of inferior parietal lobule; Electrode A: uncus, amygdala, middle temporal gyrus.

Electrode P: anterior precuneus, anterior part of inferior parietal lobule; Electrode X: cingulate gyrus (splenium), posterior precuneus, supramarginalis gyrus; Electrode M: posterior paracentral lobule, intermediate postcentral gyrus; Electrode C: parahippocampic gyrus, posterior hippocampus, posterior part of middle temporal gyrus; Electrode Z: posterior paracentral lobule, anterior part of superior parietal lobule; Electrode K: anterior precuneus, posterior part of superior parietal lobule; Electrode D: intermediate fusiform gyrus, posterior part of inferior and of middle temporal gyrus; Electrode U: intermediate part of superior temporal gyrus; Electrode W: transverse temporal gyrus, posterior part of superior temporal gyrus; Electrode S: lateral insula, central operculum.

children and should be investigated carefully by the neuroradiologist. Clinically, they are associated with early-onset severe epilepsy and are potentially amenable to curative surgical treatment, even without invasive evaluation if the electrophysiology is concordant.

6. In patients with "restricted", but multilobar, dysplasia, SEEG can be highly effective in delineating an individually-tailored cortical resection.

\section{Uncited references}

Blume and Wiebe, 2000, and Ojemann et al., 1989.

\section{Acknowledgements and disclosures.}

We thank Dr Steve Gibbs for careful reading of the manuscript and helpful comments on language. We also thank the two anonymous reviewers for their constructive criticism.

Q10 


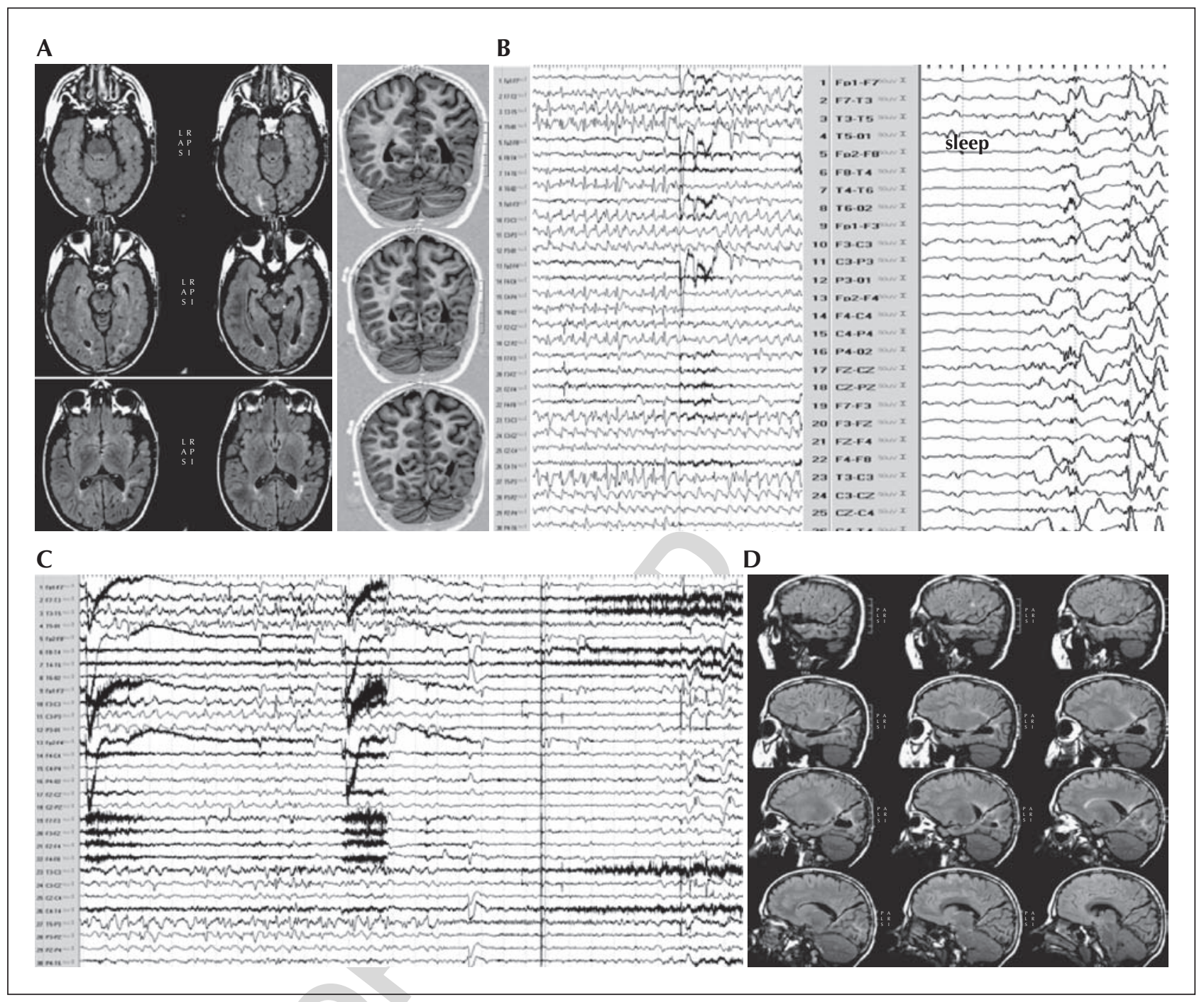

Figure 5. Patient 38. Male, born 5/1998. The patient was adopted at 2 years and 7 months of age, with no clear medical information available before this age. The patient was born before term, with normal psychomotor development, and normal neurological and neuropsychological examination. The patient was left-handed, with right-foot preference, and exhibited the presence of a slight convergent strabismus of the right eye. Seizure onset occurred at 5 years, described as "absences" and characterised by loss of contact, with diffuse hypertonia and elevation of the arms, slightly predominant to the right, followed by saccadic deviation of the eyes towards the right, with possible automatic swallowing, and no postictal deficits. Seizure frequency was 300/month. Frequently, the seizure was preceded by the patient saying "Mom, I am having a spell". A second, rare type of seizure was characterised by an isolated visual illusion (the patient saw his mother transforming into a monster) and an expression of fear.

MRI showed a smaller left hemisphere with a signal alteration of the TO region, suggesting a dysplastic or ulegyric lesion (a). Interictal EEG revealed a normal background on the right and the presence of rather subcontinuous rhythmic spikes and polyspikewaves involving the left temporo-parieto-occipital (TPO) region (b). With time, interictal discharges progressively involved the posterior right region.

Ictal EEG showed a rapid ictal discharge on the left TPO area (c). At 7 years of age, a left occipital lobectomy was performed, associated with a functional disconnection of the temporal lobe and infero-posterior area of the parietal lobe (d). Histopathological analysis revealed the presence of gliosis. The child was classified as Engel class la. Neuropsychological and psychiatric evaluation at five years post-surgery was normal, with subtle difficulties in working memory and accuracy and speed of reading. 


\section{References}

Adolphs R. Neural systems for recognizing emotion. Curr Opin Neurobiol 2002; 12: 169-77.

Alemayehu S, Bergey GK, Barry E, et al. Panic attacks as ictal manifestations of parietal lobe seizures. Epilepsia 1995; 36: 824-30.

Bancaud J. Epileptic crises of occipital origin (stereoencephalographic study). Rev Otoneuroophtalmol 1969; 41: 299-314.

Bancaud J, Angelergues R, Bernouilli C, et al. Functional stereotaxic exploration (SEEG) of epilepsy. Electroencephalogr Clin Neurophysiol 1970; 28: 85-6.

Bartolomei F, Gavaret M, Hewett R, et al. Neural networks underlying parietal lobe seizures: a quantified study from intracerebral recordings. Epilepsy Res 2011; 93: 164-76.

Bien CG, Benninger FO, Urbach H, Schramm J, Kurthen M, Elger CE. Localizing value of epileptic visual auras. Brain 2000; 123: 244-53.

Binder D, Von Lehe M, Kral T, et al. Surgical treatment of occipital lobe epilepsy. J Neurosurg 2008; 109:57-69.

Binder D, Podlogar M, Clusmann H, et al. Surgical treatment of parietal lobe epilepsy. J Neurosurg 2009; 110:1170-8.

Bleasel A, Dinner D. Mesial frontal epilepsy. In: Lüders UO. Textbook of epilepsy surgery. London: Informa healthcare, 2008: 274-84.

Blume WT, Wiebe S. Occipital lobe epilepsies. Adv Neurol 2000; 84: 173-187Q13.

Blume W, Wiebe S, Tapsell LM. Occipital epilepsy: lateral versus mesial. Brain 2005; 128: 1209-25.

Boesebeck F, Schulz R, May T, Ebner A. Lateralizing semiology predicts the seizure outcome after epilepsy surgery in the posterior cortex. Brain 2002; 125: 2320-31.

Caicoya AG, Macarron J, Albisua J, Serratosa JM. Tailored resections in occipital lobe epilepsy surgery guided by monitoring with subdural electrodes: characteristics and outcome. Epilepsy Res 2007; 77:1-10.

Cardinale F, Cossu M, Castana L, et al. Stereoelectroencephalography: surgical methology, safety and stereotactic application accuracy in 500 procedures. Neurosurgery 2013; 72: 353-66.

Colombo N, Tassi L, Galli C, et al. Focal cortical dysplasias: MRI imaging, histopathologic and clinical correlations in surgically treated patients with epilepsy. Am J Neuroradiol 2003; 24: 724-33.

Cossu M, Cardinale F, Castana L, Nobili L, Sartori I, Lo Russo G. Stereo-EEG in children. ChildsNervSyst 2006; 22: 766-78.

Cossu M, Schiariti M, Francione S, et al. Stereoencephalography in the presurgical evaluation of focal epilepsy in infancy and early childhood. J NeurosurgPediatr 2012; 9: 290-300.

D'Agostino MD, Bastos A, Piras C, Bernasconi A, et al. Posterior quadrantic dysplasia or hemi-hemimegalencephaly, A characteristic brain malformation. Neurology 2004;62: 2214-20.
Dalmagro CL, Bianchin MM, Velasco TR, et al. Clinical features of patients with posterior cortex epilepsies and predictors of surgical outcome. Epilepsia 2005; 46: 1442-9.

Dieterich M. Functional brain imaging: a window into the visuo-vestibular systems. Curr Opin Neurol 2007; 20: 12-8.

Duchowny M. Clinical, functional and neurophysiologic assessment of dysplastic cortical networks: implications for cortical functioning and surgical management. Epilepsia 2009; 50: 19-27.

Engel J, Van Ness P, Rasmussen T, Ojemann L. Outcome with respect to epileptic seizures. In: Surgical treatment of the epilepsies. $2^{\text {nd }}$ edition. Engel Jr J. New York : Raven Press, 1993: 609-21.

Fogarasi A, Boesebeck F, Tuxhorn I. A detailed analysis of symptomatic posterior cortex seizure semeiology in children younger than seven years. Epilepsia 2003; 44: 89-96.

Fogarasi A, Tuxhorn I, Hegyi M, Janszky J. Predictive clinical factors for the differential diagnosis of childhood extratemporal seizures. Epilepsia 2005; 46: 1280-5.

Gil-Nagel A, Garcia Morales I, HimenezHuete A, et al. Occipital lobe epilepsy secondary to ulegyria. J Neurol 2005; 252: 1178-85.

Godoy J, Lüders H, Dinner D, Morris H, Wyllie E. Versive eye movements elicited by cortical stimulation of the human brain. Neurology 1990; 40: 296-9.

Ibrahim GM, Fallah A, Albert GW, et al. Occipital lobe epilepsy in children: characterization, evaluation and surgical outcomes. Epilepsy Res 2012; 93:335-45.

Jehi L, O'Dwyer R, Najm I, Alexopoulos A, Bingaman W. A longitudinal study of surgical outcome and its determinants following posterior cortex epilepsy surgery. Epilepsia 2009; 50: 2040-52.

Jobst B, Williamson $\mathrm{P}$, Thadani $\mathrm{V}$, et al. Intractable occipital lobe epilepsy: Clinical characteristics and surgical treatment. Epilepsia 2010; 51:2334-7.

Kahane P, Hoffmann D, Minotti L, et al. Reappraisal of the human vestibular cortex by cortical electrical stimulation study. Ann Neurol 2003; 54: 615-24.

Kahane P, Landre E, Minotti L, Francione S, Ryvlin P. The Bancaud and Talairach view on the epileptogenic zone: a working hypothesis. Epileptic Disord 2006; 8: S16-26.

Kellinghaus C, Skidmore C, Loddenkemper T. Lateralizing value of epileptic nystagmus. Epilepsy Behav 2008; 13: 700-2.

Kim $\mathrm{CH}$, Chung $\mathrm{CK}$, Lee SK, Lee YK, Chi JG. Parietal lobe epilepsy: surgical treatment and outcome. Stereotact Funct Neurosurg 2004a; 82: 175-85.

Kim DW, Lee SK, Yun $\mathrm{CH}$, et al. Parietal lobe epilepsy: the semiology, yield of diagnostic workup, and surgical outcome. Epilepsia 2004b; 45: 641-9.

Lee HW, Hong SB, Seo DW, Tae WS, Hong SC. Mapping of functional organization in human visual cortex: Electrical cortical stimulation. Neurology 2000; 54: 849-84.

Liava A, Francione $\mathrm{S}$, Cossu $\mathrm{M}$, et al. Individually tailored extratemporal lobe epilepsy surgery in children: anatomo-electro-clinical features and outcome predictors in a population of 53 cases. Epilepsy Behav 2012; 25: 68-80. 
Ludwig BI, Marsan CA. Clinical ictal patterns in epileptic patients with occipital electroencephalographic foci. Neurology $1975 ; 25: 463-71$.

Manford M, Fish DR, Shorvon SD. An analysis of clinical seizure pattern and their localizing value in frontal and temporal lobe epilepsies. Brain 1996; 119: 17-40.

Mohamed A, Freeman J, Maixner V, Bailey V, Wrennall J, Harvey $A$. Temporoparietooccipital disconnection in children with intractable epilepsy. J NeurosurgPediatrics 2011; 7:66070 .

Munari C, Bonis A, Kochen S, et al. Eye movements and occipital seizures in man. Acta Neurochirurgica Suppl 1984; 33: 47-52.

Munari C, Tassi L, Francione S, Kahane P, Malapani C, Lo Russo G, Hoffmann D. Occipital seizures with childhood onset in severe partial epilepsy: a surgical perspective. In: Andermann F, Beaumanoir A, Mira L, Roger J, Tassinari CA. Occipital Seizures and Epilepsies in Children. London: John Libbey and Company Ltd.,1993: 203-11.

Ojemann GA, Ojemann J, Lettich E, et al. Cortical language localization in left dominant hemisphere. J Neurosurg 1989; 71:316Q14.

Palmini A, Andermann F, Dubeau F, et al. Occipitotemporal epilepsies: evaluation of selected patients requiring depth electrodes studies and rationale for surgical approaches. Epilepsia 1993; 34: 84-96.

Penfield W, Jasper H. Epilepsy and the functional anatomy of the human brain. Boston: Little, Brown and Co, 1954.
Pierrot-Deseilligny C, Milea D, Muri RM. Eye movement control by the cerebral cortex. Curr Opin Neurol 2004;17: 17-25.

Ristic A, Alexopoulos A, So N, Wong C, Najm I. Parietal lobe epilepsy: the great imitator among focal epilepsies. Epileptic Disord 2012; 14: 22-31. doi: 10.1684/epd.2012.0484.

Salanova V, Andermann F, Olivier A, Rasmussen T, Quesney LF. Occipital lobe epilepsy: electroclinical manifestations, electrocorticography, cortical stimulation and outcome in 42 patients treated between 1930 and 1991. Surgery of occipital lobe epilepsy. Brain 1992; 115: 1655-80.

Salanova V, Andermann F, Rasmussen T, Olivier A, Quesney LF. Parietal lobe epilepsy. Clinical manifestations and outcome in 82 patients treated surgically between 1929 and 1988. Brain 1995; 118: 607-27.

Takeda A, Bancaud J, Talairach J, Bonis A, Bordas-Ferrer M. Concerning epileptic attacks of occipital origin. Electroencephal Clin Neurophysiol 1970; 28: 647-8.

Tandon N, AlexopoulosA, Warbel A, Najm I, Bingaman W. Occipital epilepsy: spatial categorization and surgical management. J Neurosurg 2009; 110:306-18.

Thier P, Andersen RA. Electrical microstimulation distinguishes distinct saccade-related areas in the posterior parietal cortex. J Neurophysiol 1998; 80:1713-35.

Williamson PD, Thadani VM, Darcey TM, Spencer DD, Spencer SS, Mattson RH. Occipital lobe epilepsy: clinical characteristics, seizure spread patterns, and results of surgery. Ann Neurol 1992; 31:3-13.

\section{Supplementary tables}

\section{Detailed anatomo-electro-clinical features of each patient}

N: neurological; Npsy: neuropsychological; Psy: psychiatric; yrs: years; rdf: fundamental rhythm; op: operative; FU: follow-up (Engel classification); n: normal; m: medium; s: severe; O: occipital; T: temporal; C: central; P: parietal; F: frontal; G: generalisation; L: left; R: right; EVH: elementary visual hallucinations; CVH: complex visual hallucinations; am: amaurosis; VI: visual illusions; E: eye; $\mathrm{H}$ : head; EH: eye-head; dev: deviation; ipsi: ipsilateral; contr: contralateral; lat: lateralised; gy: oculogyric movements; ny: epileptic nystagmus; as: asymmetric; s: symmetric; na: non available; Hy: focal hypertonia; Dy: focal contralateral dystonia; autom: automatisms; mastic: mastication; rot: rotation; $\mathrm{HH}$ : homonymous hemianopsia; HQ: homonymous quadrantanopsia; hemi-hemimeg: hemi-hemimegalencephaly; hipp: hippocampus; parahipp: parahippocampic; TSC: tuberous sclerosis complex; SW: Sturge Weber; ${ }^{2} 2$-step operation; pf: partial resection of the epileptogenic zone; Dt: disconnection; Lp: Q11;ESES: Q12. 
A. Liava, et al.

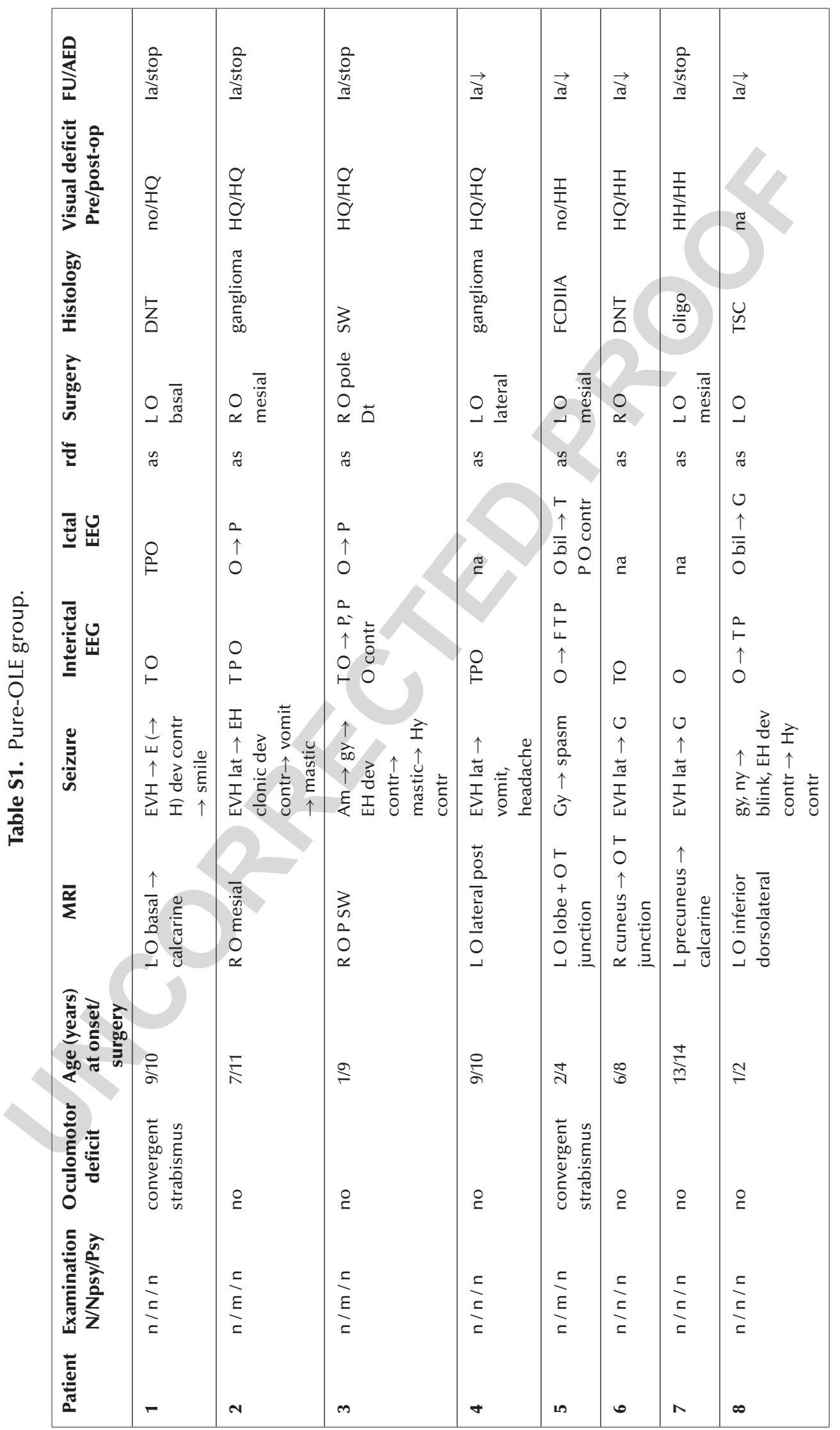




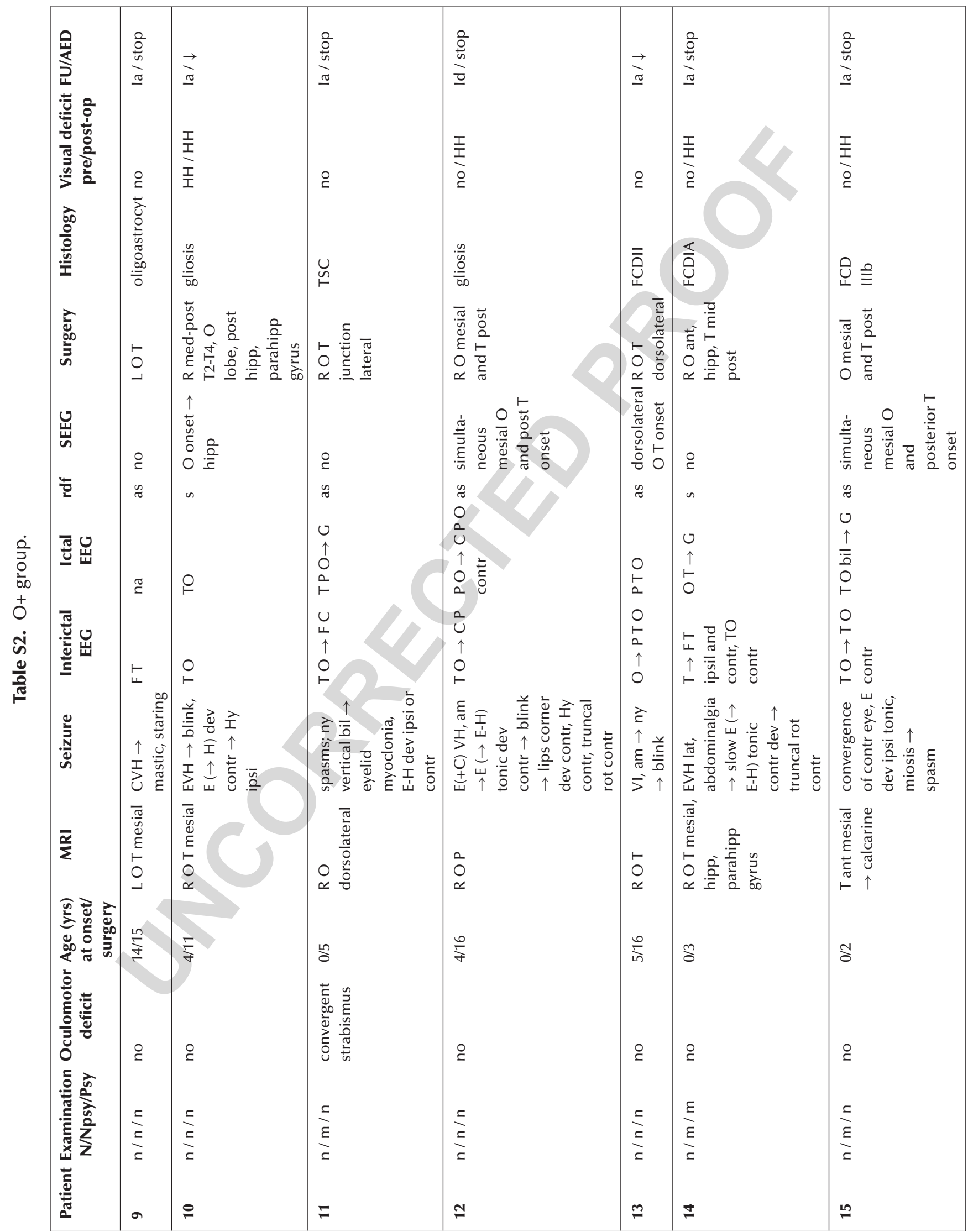


A. Liava, et al.

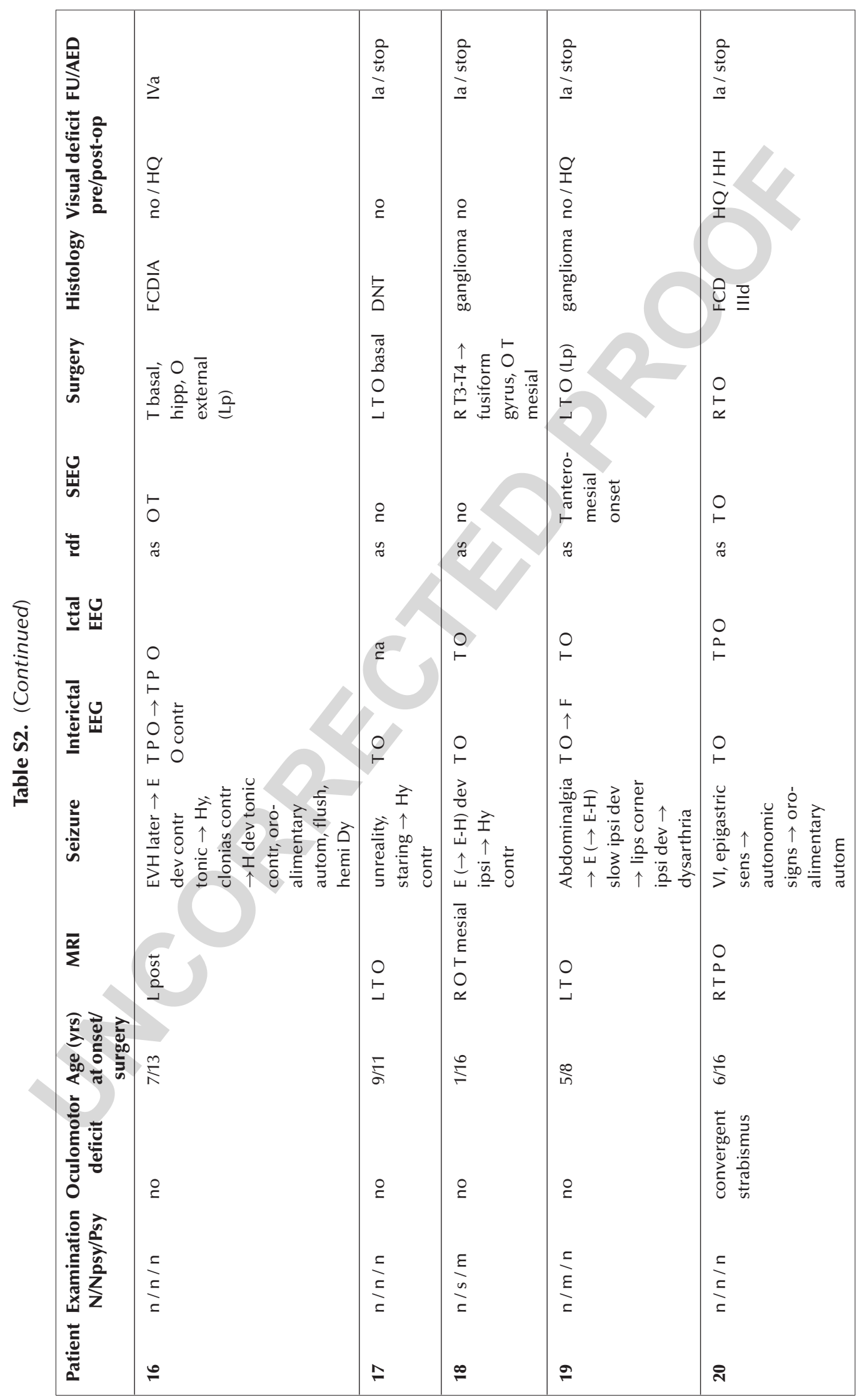




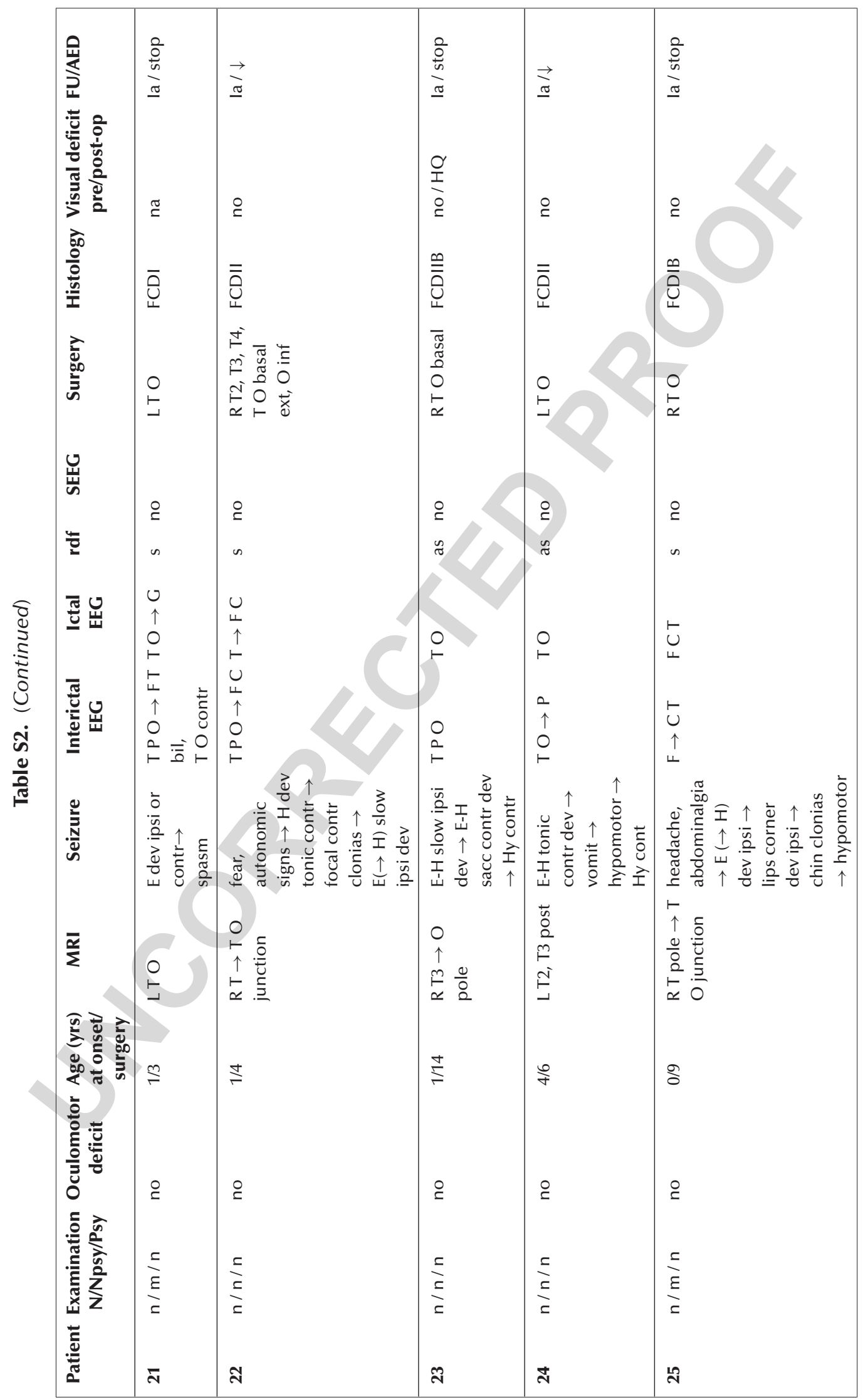


A. Liava, et al.

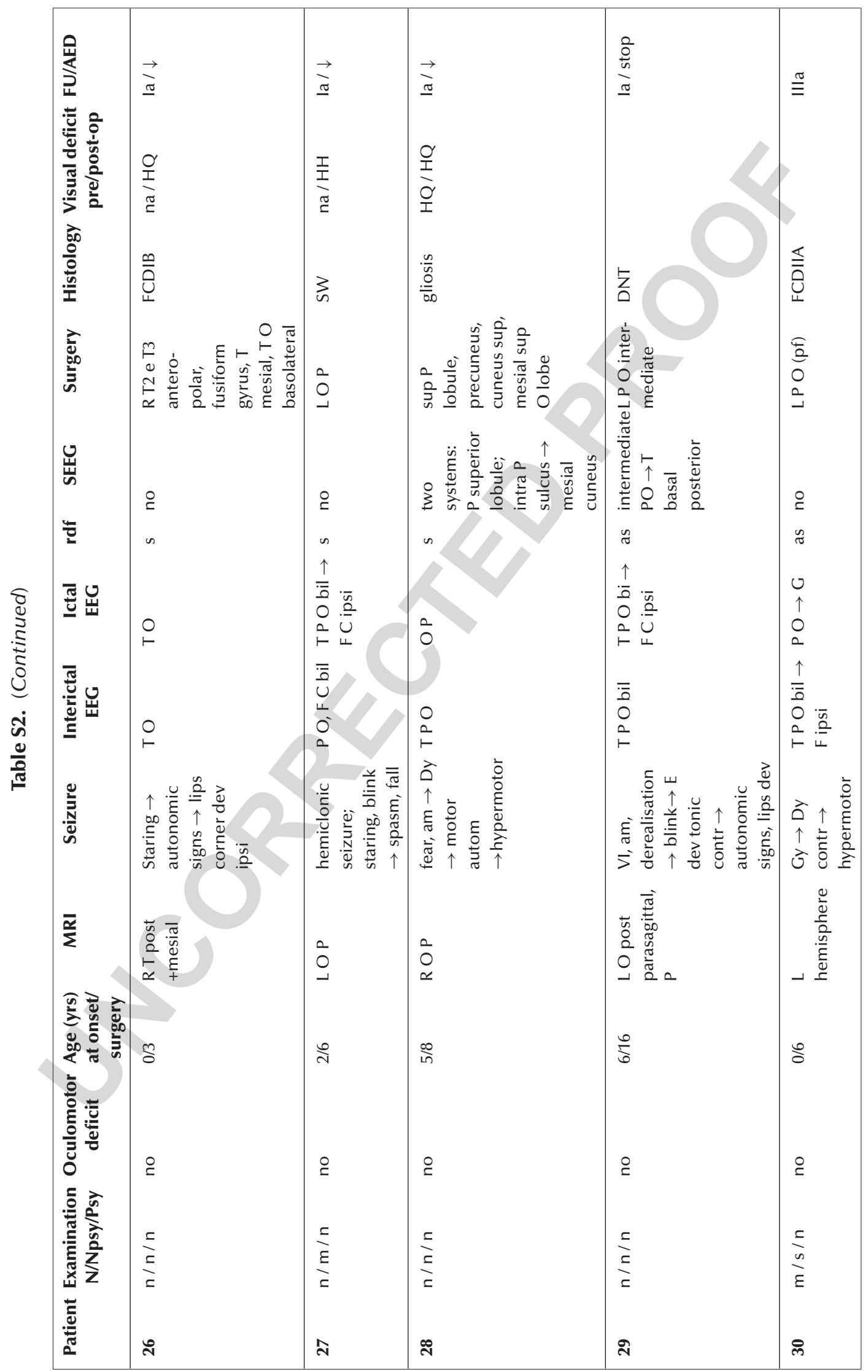




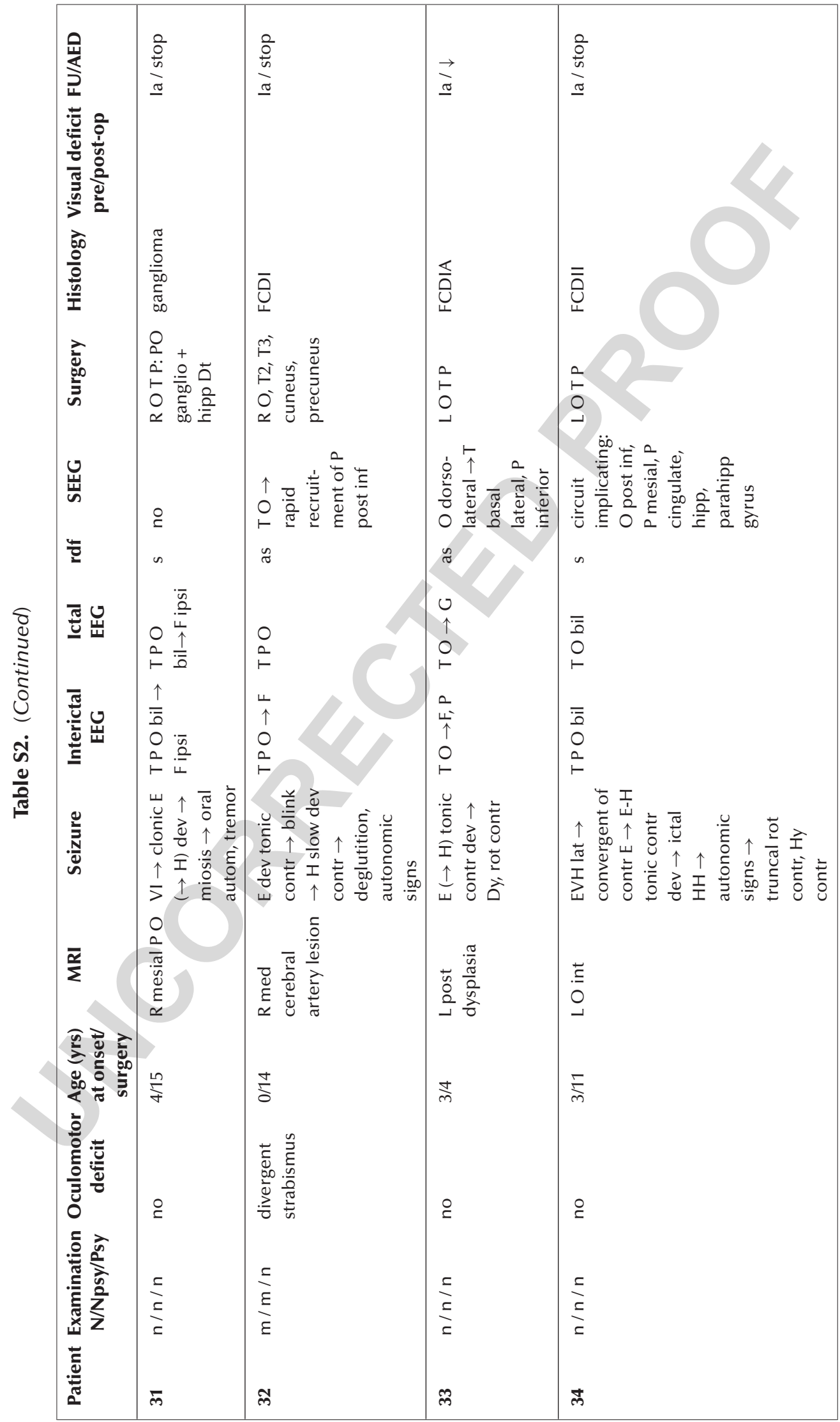


A. Liava, et al.

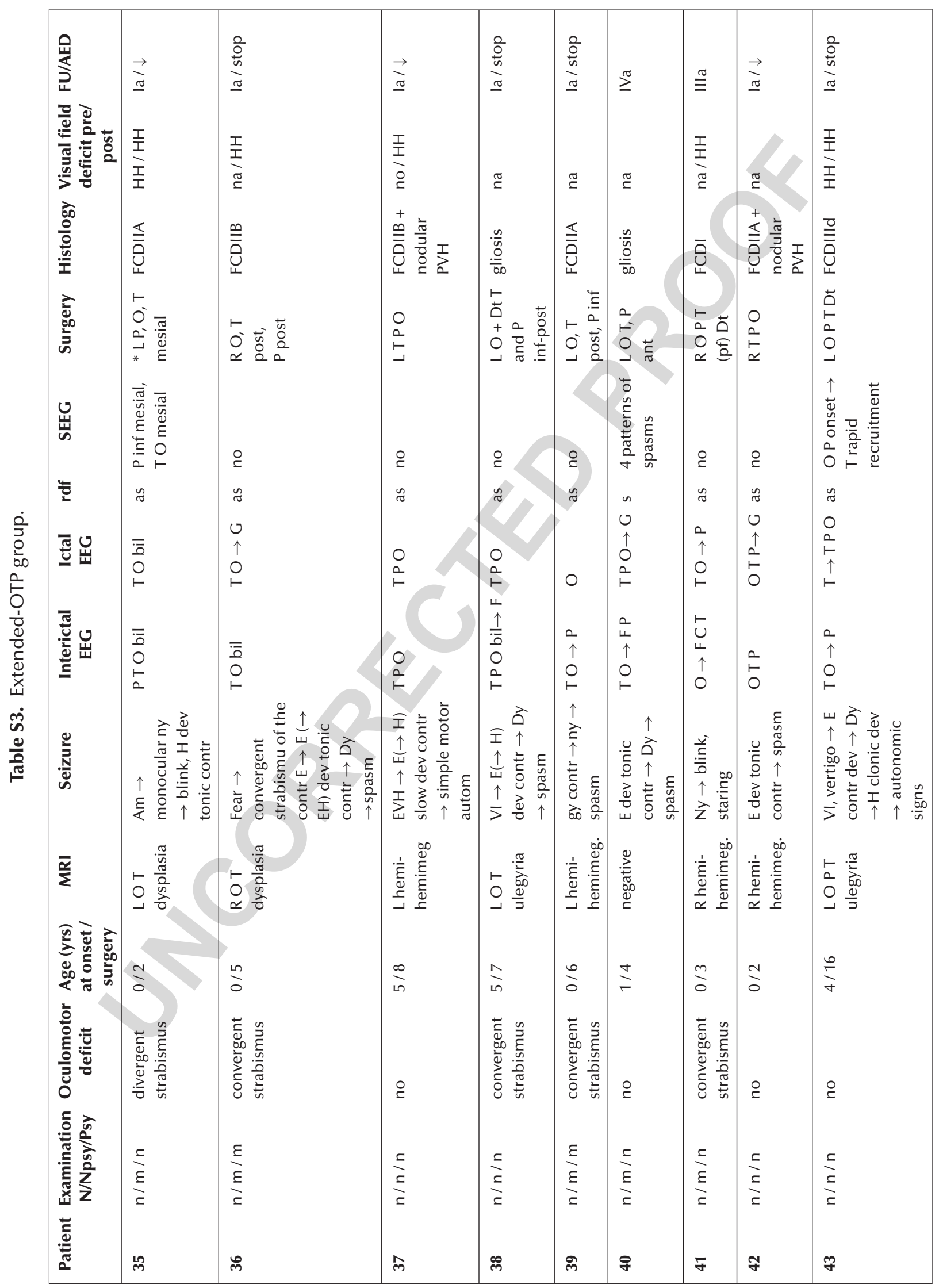




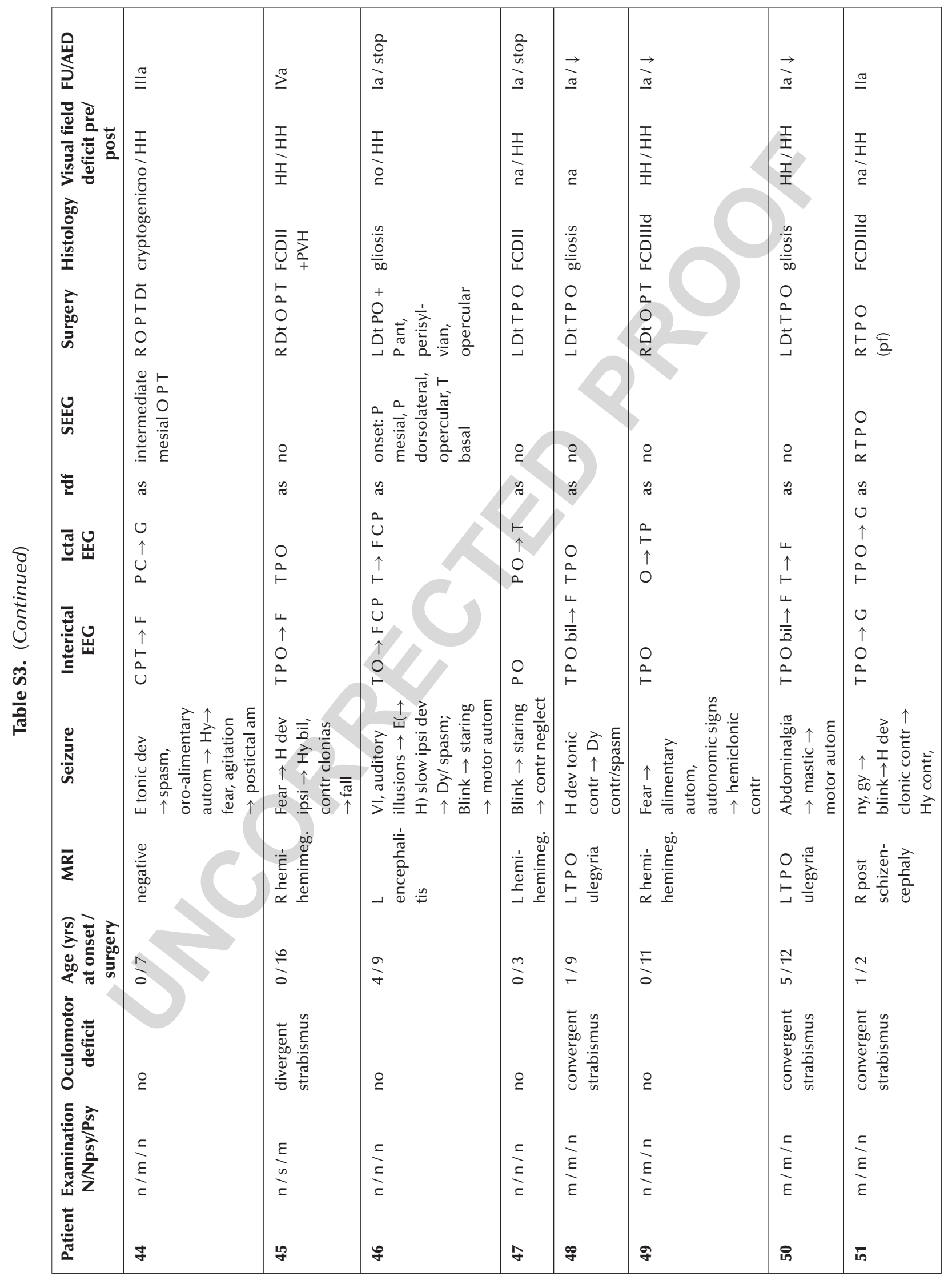


A. Liava, et al.

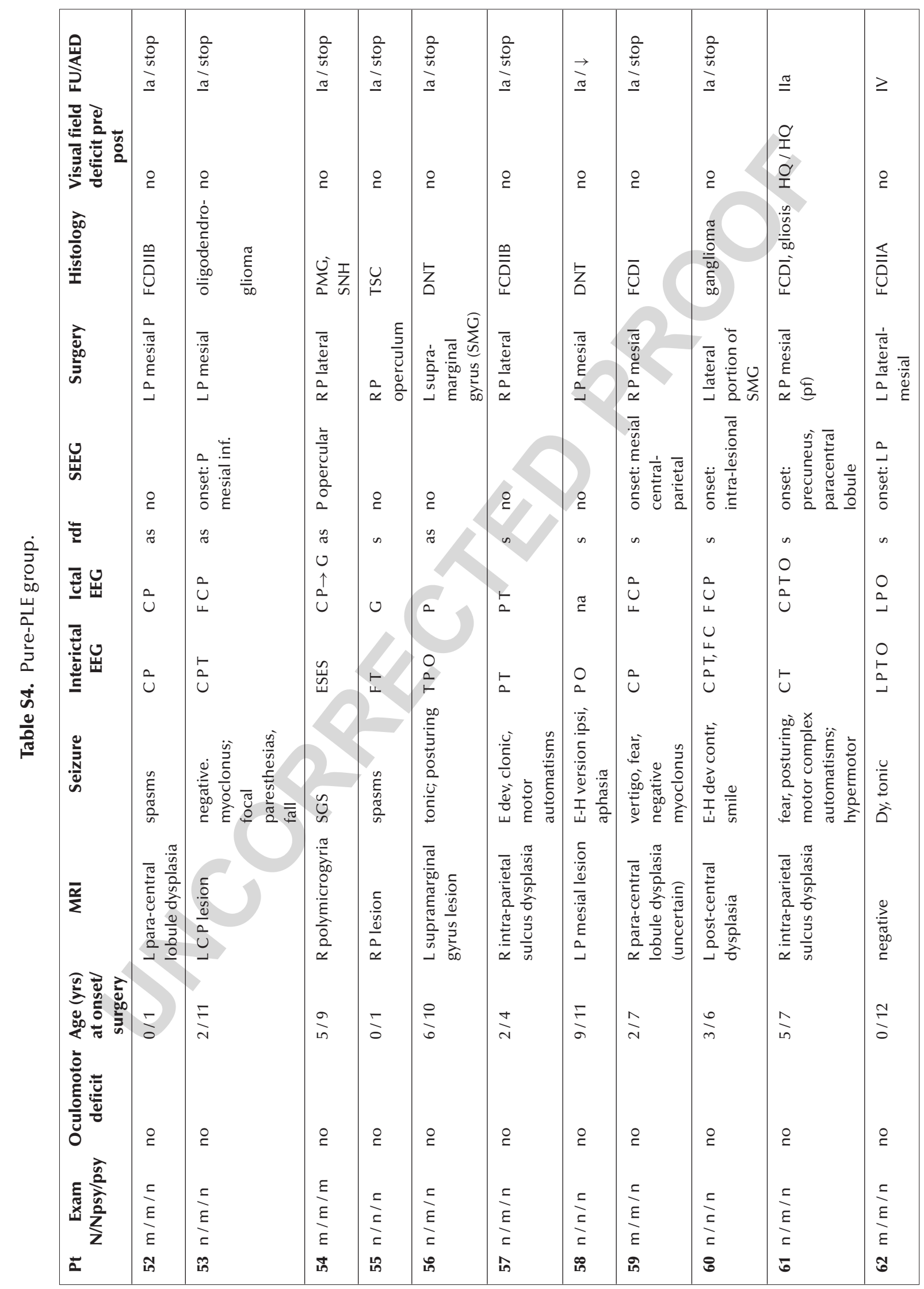




\section{Author Queries}

Q1 reference not in list; please provide the reference or delete from text

Q2 reference not in list; please provide the reference or delete from text

Q3 reference not in list; please provide the reference or delete from text

Q4 reference not in list; please provide the reference or delete from text

Q5 reference not in list; please provide the reference or delete from text

Q6 reference not in list; please provide the reference or delete from text

Q7 reference not in list; please provide the reference or delete from text

Q8 which of $\mathrm{a} / \mathrm{b}$ ?

Q9 reference not in list; please provide the reference or delete from text

Q10 Please provide the appropriate statements relative to conflicts of interests

Q11 ??

Q12 ??

Q13 Reference not in text; please provide the appropriate callout or delete from list

Q14 Reference not in text; please provide the appropriate callout or delete from list 